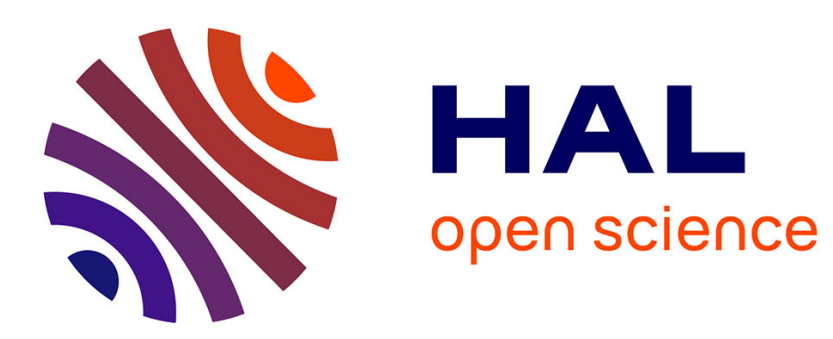

\title{
Vibrational properties of quasi-periodic beam structures
} Arthur Glacet, Anne Tanguy, Julien Réthoré

\section{To cite this version:}

Arthur Glacet, Anne Tanguy, Julien Réthoré. Vibrational properties of quasi-periodic beam structures. Journal of Sound and Vibration, 2019, 10.1016/j.jsv.2018.10.045 . hal-03360640

\section{HAL Id: hal-03360640 https://hal.science/hal-03360640}

Submitted on 30 Sep 2021

HAL is a multi-disciplinary open access archive for the deposit and dissemination of scientific research documents, whether they are published or not. The documents may come from teaching and research institutions in France or abroad, or from public or private research centers.
L'archive ouverte pluridisciplinaire HAL, est destinée au dépôt et à la diffusion de documents scientifiques de niveau recherche, publiés ou non, émanant des établissements d'enseignement et de recherche français ou étrangers, des laboratoires publics ou privés. 


\title{
Vibrational properties of quasi-periodic beam structures
}

\author{
Arthur Glacet, Anne Tanguy, Julien Réthoré ${ }^{1}$ \\ LaMCoS, Université de Lyon / INSA Lyon / CNRS UMR 5259 \\ Bat. Jacquard, 27 Avenue Jean Capelle, F-69621 Villeurbanne, Cedex, France
}

\begin{abstract}
Quasi-periodic structures have been widely studied, notably in the atomic vibration domain. In this paper a beam structure based on Octagonal quasi-periodic tiling is studied. We provide a complete description of its vibrational response, including the density of its vibrational states, a detailed description of its vibration modes, and the computation of the dynamical structure factor (spectral density of energy) for transverse and for longitudinal waves. It is shown that quasi-periodic structures exhibit localized low frequency vibration modes that are due to resonant vibrations of isolated patterns in the quasi-periodic structure, but in opposite, high-frequency modes are (non-trivially) extended. Moreover, the paper shows the possible existence of band gaps in the vibrational response of periodic and quasi-periodic beam lattices as a function of the ratio between the bending and the tensile stiffness of the beams.
\end{abstract}

Keywords: Quasi-periodic, Band Gap, Vibration, Beam Lattice, Dispersion Relation

\section{Introduction}

Quasi-periodic materials have been widely studied for their atomic dynamics, photonic, magnetic and electronic wave propagation [1, 2, 3]. These structures exhibit complex vibrational behavior, including a set of frequency ranges in which no propagative wave ex-

5 ist [4, 5], i.e. band gaps. Band gaps can lead to interesting applications in various domains [6]. The recent progress in additive manufacturing open new possibilities for building large scale quasi-periodic structures by allowing the printing of complex metamaterials, in a consistent manner. Additive manufactured metamaterials can be designed to exhibit unusual macroscopic behavior due to their internal structure as in [7, 8, 9]. Therefore the possibility of creating metamaterials having the same properties as the quasi-periodic atomic structures can be highly interesting and allows to get rid of unwanted complex behaviors [5]. Such metamaterials could create band gaps in their vibrational mechanical response while being isotropic regarding elasticity or wave propagation for example. Moreover, the macroscopic beam structure offers additional possibilities in terms of large scale interactions and control parameters for tuning the vibrational properties.

The mechanical and vibrational properties of quasi-periodic and of amorphous structures are related to complex mathematical problems due to the impossibility of periodic simplifications. Therefore, in order to solve these problems, big size matrix problems have to be dealt with. Moreover, usual Fourier transform-based computational tools, or Bloch 2o Wave expansions, that are very interesting for periodic structures [10] would be very unefficient for such systems. The numerical approaches followed in this paper deal with

Email addresses: arthur.glacet@insa-lyon.fr (Arthur Glacet), anne.tanguy@insa-lyon.fr (Anne Tanguy), julien.rethore@ec-nantes.fr (Julien Réthoré)

${ }^{1}$ Now at GEM, CNRS UMR 6183 CNRS / Ecole Centrale de Nantes / Université de Nantes 
completely resolved calculations on very large matrices, thus avoiding the required simplifications associated to (even highly and recently elaborated) homogeneization tools as in [11]. In the later case, the degree of spatial resolution that depends on the waves frequency is indeed crucial for an accurate description, as well as the hypotheses concerning the order of expansion of the constitutive laws in the different order parameters (displacements and rotations of beam nodes for example) [12]. In contrary, our method allows for direct insights into the dynamical behaviour at any vibrational frequency of non-periodic systems, including the description of possible localized vibrations that are difficult to identify and to take into account in homogeneization procedures [13, 14, 15]. The Octagonal Quasiperiodic tiling is chosen in this paper for its ability to create a periodic approximant to the quasi-periodic tiling thus allowing the use of periodic boundary conditions as suggested in [16]. It has been shown in [17] that, for ferromagnetic properties, the approximant with periodic boundary conditions closely mimics the infinite lattice properties. The numeri-

35 cal methods used here for the vibrational study of big systems were inspired from atomic vibration analyses and adapted to finite element modeling of large scale complex beam structures. First, the vibrational eigenmodes are computed by exact diagonalization of the dynamical matrix restricted to the beam nodes. Then, Kernel Polynomial Method (KPM) is used to calculate the complete Vibrational Density Of States (VDOS) and the Dynamical Structure Factor (DSF), giving rise to the complete dispersion relation without the need of exact diagonalization of the dynamical matrix. The KPM method is detailed by [18] and was adapted recently to the study of the vibrational properties of large-size atomic systems by [19]. We apply it here to large scale beam structures. This method allows accurate description of the vibrational properties of large scale systems, and thus a better understanding

45 of the vibrational response of quasi-periodic structures. Thanks to this work, it will now be easy to transfer this method to the detailed vibrational study of any beam structure.

The paper is organized as follows: first the modeling and numerical methods are explained in Section 2 and 3 . These methods are firstly applied on a simple periodic beam structure in Section 4 in order to be validated, and to show the influence of the bending stiffness on the vibrational response. Then in Section 5 the methods are applied to the complete analysis of the vibrational response of an Octagonal quasi-periodic approximant, including the detailed analysis of its eigenmodes.

\section{Model}

The beam structure is described by a Finite Element (FE) model based on Euler-Bernoulli beam theory. In this description, each of the $N$ nodes of the model holds 3 degrees of freedom (DF), the two components $\left(u_{i}, v_{i}\right)$ of the displacement, and $\theta_{i}$ the rotation of the beam section at the node $i$ (Fig. 11). The dynamical problem can be written in terms of nodal DF with classical matrix equation:

$$
\mathbf{K d}+\mathbf{M} \ddot{\mathbf{d}}=\mathbf{F},
$$

where $\mathbf{d}$ is the DF vector containing all DFs of all nodes, $\mathbf{K}$ the stiffness matrix, $\mathbf{M}$ the mass matrix and $\mathbf{F}$ the external loads in all directions $\left(F_{u}, F_{v}\right.$ and $\left.M_{z}\right)$ for all nodes: $F_{u}$ and $F_{v}$ are the components of the external load vector along and perpendicular to the beam, and $M_{z}$ is the torque perpendicular to the structure's plane (Fig. 11).

The stiffness and mass matrix are obtained first for each element by using the weak formulation of the continuous beam problem written on the two extremities of the beam [20]. For readers unused to the Euler-Bernouilli beam theory, we recall here the expression of the corresponding elementary stiffness matrix $\mathbf{K}_{\mathbf{e}}$ and the elementary mass matrix $\mathbf{M}_{\mathbf{e}}$ written in the local element basis and using the elementary vector of degrees of freedom 7o $\left\{u_{1}, v_{1}, \theta_{1}, u_{2}, v_{2}, \theta_{2}\right\}$ ( $u_{1}$ displacement along the direction defined by the beam at node 1 , 
$v_{1}$ displacement orthogonal to the beam orientation at node $1, \theta_{1}$ rotation of node $\left.1 \ldots\right)$ [21]:

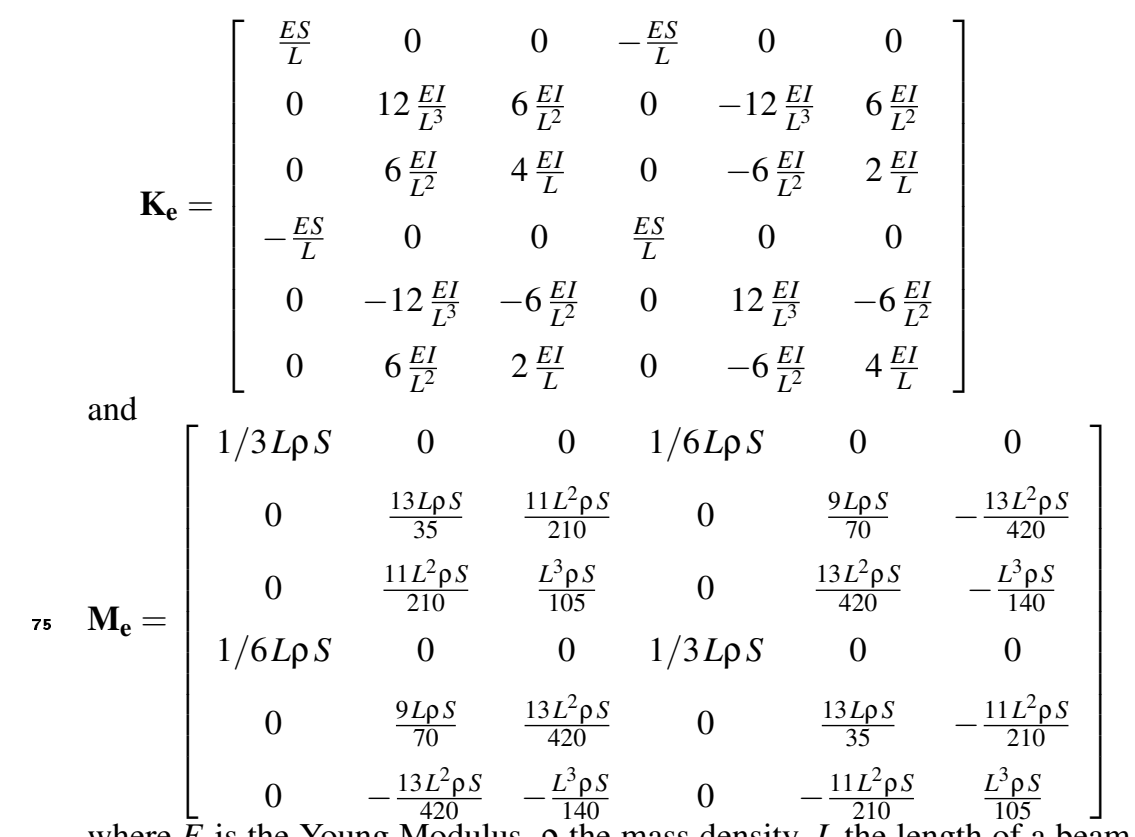

where $E$ is the Young Modulus, $\rho$ the mass density, $L$ the length of a beam element, $S$ the area of a beam section and $I$ the quadratic moment of a beam section. Those elementary matrices are then transfered in the global basis by a local change of coordinates (rotation) and summed over all the elements to get the global matrix involved in 1

80

When studying the vibrational response, the external forces on each node are zero because the system would be at equilibrium at rest, and the displacement is assumed to be a wave solution, that is:

$$
\ddot{\mathbf{d}}=-\omega^{2} \mathbf{d},
$$

where $\omega$ is the angular frequency of the wave. Thus the dynamical problem can be written

$$
\mathbf{K d}=\omega^{2} \mathbf{M d} .
$$

85 The periodic boundary conditions are imposed by modifying the previous equation thus equating the DFs of the homologous nodes. $M$ being positive and symetric, it can be written $\mathbf{M}=\mathbf{L} \mathbf{L}^{\mathbf{t}}$. The problem thus becomes a classical eigenmodes problem

$$
\mathbf{H d}^{\prime}=\lambda \mathbf{d}^{\prime}
$$

with

$$
\mathbf{H}=\mathbf{L}^{-1} \mathbf{K L}^{-\mathbf{t}} ; \lambda=\omega^{2} ; \mathbf{d}^{\prime}=\mathbf{L}^{\mathbf{t}} \mathbf{d},
$$

where $\mathbf{H}$ is a symmetric positive definite square matrix. This dynamical matrix depends on three parameters:

90

$$
\begin{aligned}
& K_{v}=12 \frac{E I}{L^{3}} \text { the flexural stiffness. } \\
& K_{u}=\frac{E S}{L} \text { the traction/compression stiffness. } \\
& m=\rho S L \text { the mass of a beam element. }
\end{aligned}
$$

Note that the ratio $\frac{K v}{K u}$ that will be discussed later, depends here only on the geometry of the beam cross section and on its length. 
Moreover, the discretization of the system on the nodes at the extremities of the beams does not allow getting insight on vibrations at a scale smaller than half of the smallest beam length, thus limiting the highest frequency reached. The highest vibrational frequency $\omega_{\max }$ is of the order of the highest possible frequency supported by the smallest element, that is given by solving the equation $\operatorname{det}\left(\mathbf{K}_{\mathbf{e}}-\omega^{2} \mathbf{M}_{\mathbf{e}}\right)=\mathbf{0}$ and depends on the aspect ratio of the beams. Due to the very large number of nodes, we will solve Eq. 3 with numerical methods that are adapted to very large system sizes. We will compare the exact diagonalization of the matrix $\mathbf{H}$ (allowing exact identification of the modes but only for $N \lesssim 10000$ ) to the recursive calculation of the frequency density (VDOS) and of the dispersion relation (DSF) that will be presented later and that is not size limited.

\section{Numerical Methods}

\subsection{Exact diagonalization}

The exact diagonalization of the dynamical matrix $\mathbf{H}$ allows getting directly $\mathbf{d}^{\prime}$ from Eq. 3, and thus the DF vector $\mathbf{d}=\mathbf{L}^{-\mathbf{t}} \mathbf{d}^{\prime}$. The diagonalization is performed using the build in function eigsn in Matlab software. The calculation is limited by the system size. For sufficienlty small systems $(N \lesssim 10000)$, it allows visualizing the $3 \times N$ eigenvectors that are the resonant modes of the system (see Section 5). It allows also for computing the participation ratio (PR) of each mode. For a given eigenmode $j$, the PR gives information on the ratio of particles participating in each vibration mode. It was used for example to identify possible localized vibrations in disordered systems [22, 23, 24, 25]. It is defined as:

$$
P R\left(\omega_{j}\right)=\frac{1}{N} \frac{\left(\sum_{i}\left|\mathbf{u}_{\mathbf{i}}\right|^{\mathbf{2}}\left(\omega_{\mathbf{j}}\right)\right)^{\mathbf{2}}}{\sum_{i}\left|\mathbf{u}_{\mathbf{i}}\right|^{\mathbf{4}}\left(\omega_{\mathbf{j}}\right)}
$$

where $\mathbf{u}_{\mathbf{i}}$ is the displacement vector $\mathbf{u}_{\mathbf{i}}=\left\{\mathbf{u}_{\mathbf{x}_{\mathbf{i}}}, \mathbf{u}_{\mathbf{y}_{\mathbf{i}}}\right\}$ for the $i$ th node and $\omega_{j}$ the pulsation of the $j$ th eigenmode. It means that $P R=1 / N$ when only one isolated node over $N$ supports the vibration, while $P R=N / N=1=100 \%$ in case of uniform translation.

The determination of the eigenfrequencies and eigenmodes from the resolution of Equation 5 is highly computationaly demanding, especially for non-periodic systems. For this reason, approximate methods that do not require exact resolution of the eigenvalue problem have been developed [26, 18]. The computation of several quantities like the vibrational density of states and the dynamical structure factor (spectral densiy of states) is useful for analyzing the vibrational properties of a material. They are obtained without solving the eigenvalue problem as detailed below.

\subsection{Vibrational Density of States}

The VDOS corresponds to the distribution of the eigenfrequencies resulting from the spectral analysis of the model. It is defined as:

$$
\operatorname{VDOS}(\omega)=\frac{1}{3 N} \sum_{j=1}^{3 N} \delta\left(\omega-\omega_{j}\right)
$$

where $\delta$ is the Dirac function. Using the Kernel Polynomial Method (KPM), the VDOS can be obtained without the exact resolution of the eigenvalue problem (4). The KPM thus allows to compute the VDOS even for very large systems [26, 18]. It is based on the approximation of the $\delta$-function in Eq. 8 by a series of Tchebychev polynomials, yielding to an exact expression of the distribution of the eigenvalues without calculating the eigenvalues itself. The method is detailed in [26, 18]. The starting point is the expansion of the $\delta$-function as

$$
\delta\left(\varepsilon-\varepsilon_{j}\right)=\frac{2}{\pi} \sqrt{1-\varepsilon^{2}} \sum_{b=0}^{\infty} T_{b}(\varepsilon) T_{b}\left(\varepsilon_{j}\right) .
$$


where $\varepsilon_{j}=2 \omega_{j}^{2} / \omega_{\max }^{2}-1 . \omega_{\max }$ is the maximum frequency supported by the individual beams: it is given by the resolution of the equation $\operatorname{det}\left(\mathbf{K}_{\mathbf{e}}-\omega^{2} \mathbf{M}_{\mathbf{e}}\right)=0$, thus $-1<\varepsilon_{j}<1$, $T_{b}$ are Tchebychev polynomials that can be obtained either by the recurrence relation

$$
\begin{aligned}
& T_{0}(\varepsilon)=1 \\
& T_{1}(\varepsilon)=2 \varepsilon, \\
& T_{b}(\varepsilon)=2 \varepsilon T_{b-1}(\varepsilon)-T_{b-2}(\varepsilon)
\end{aligned}
$$

or by a trigonometric definition:

$$
T_{b}(\varepsilon)=\frac{\sin ((b+1) \arccos (\varepsilon))}{\sqrt{1-\varepsilon^{2}}} .
$$

The VDOS can thus be rewritten as

$$
\operatorname{VDOS}(\omega) \approx \frac{8 \omega}{3 N \pi \omega_{\max }^{2}} \sum_{j=1}^{3 N} \sum_{b=0}^{B} \gamma_{b} T_{b}\left(\varepsilon_{j}\right) T_{b}(\varepsilon)
$$

where $\gamma_{b}$ are Jackson's damping coefficients [27] introduced to avoid Gibbs oscillations

$$
\gamma_{b}=\frac{(B+1-b) \cos \frac{\pi b}{B+1}+\sin \frac{\pi b}{B+1} \cot \frac{\pi}{B+1}}{B+1}
$$

and $B$ is a maximum number controlled by the desired accuracy of the calculations, the approximate solution converging to the continuous solution $\operatorname{VDOS}(\omega)$ when $B$ goes to infinity [18]. Here we chose $B=300$. The Tchebychev momenta are defined as

$$
\mu_{b}=\frac{1}{3 N} \sum_{j=1}^{3 N} T_{b}\left(\varepsilon_{j}\right)=\frac{1}{3 N} \sum_{j=1}^{3 N}\left\langle\mathbf{u}_{\mathbf{j}}\left|T_{b}\left(\mathbf{H}_{\mathbf{t}}\right)\right| \mathbf{u}_{\mathbf{j}}\right\rangle
$$

with $\mathbf{H}_{\mathbf{t}}=\frac{2 \mathbf{H}}{\omega_{\max }^{2}}-\mathbf{I}$. The $\mathrm{b}^{\text {th }}$ momenta can be approximated as

$$
\mu_{b} \approx \overline{\left\langle\mathbf{d}_{0}^{r} \mid \mathbf{d}_{b}^{r}\right\rangle}
$$

where $\left|\mathbf{d}_{0}^{r}\right\rangle$ is a $3 N$ Gaussian random vector with unit norm, the upper bar is the average over $R$ random realizations of this random vector, and $\left|\mathbf{d}_{b}^{r}\right\rangle$ follows the recurrence relations

$$
\begin{aligned}
\left|\mathbf{d}_{1}^{r}\right\rangle & =2 \mathbf{H}_{\mathbf{t}}\left|\mathbf{d}_{0}^{r}\right\rangle, \\
\left|\mathbf{d}_{b}^{r}\right\rangle & =2 \mathbf{H}_{\mathbf{t}}\left|\mathbf{d}_{b-1}^{r}\right\rangle-\left|\mathbf{d}_{b-2}^{r}\right\rangle=T_{b}\left(\mathbf{H}_{\mathbf{t}}\right)\left|\mathbf{d}_{0}^{r}\right\rangle
\end{aligned}
$$

When $R$ is large enough, the variability due to the random generation of the $\left\{d_{0}^{r}\right\}$ can be neglected [26]. We used $R=30$. Using Eqs 13|14, the VDOS can thus be computed as

$$
\operatorname{VDOS}(\omega)=\frac{4 \omega}{3 N \omega_{\max }^{2}} \sum_{b=0}^{B} \gamma_{b} \mu_{b} \sin \left(2(b+1) \arcsin \left(\omega / \omega_{\max }\right)\right)
$$

that is a function of the angular frequency $\omega$ only, without the need of $\omega_{j}$ neither of $\mathbf{u}_{\mathbf{j}}$.

\subsection{Dynamical Structure Factor}

The DSF is also called Spectral Density of Energy [28]. It corresponds schematically to the combined spatial and temporal Fourier transforms of the displacements inside the system, thus giving the amplitude of the harmonic waves as a function of $q$ the wave vector and $\omega$ the angular frequency. In atomistic samples, it is related to the cross-section of photons that are scattered by atomic vibrations in non-elastic x-ray or neutron scattering experiments [29]. For longitudinal modes for example, it is related to the spatial correlation 
function of density fluctuations. As an indicator of the connection between wave-vectors and frequency during the dynamical response of the system, the DSF can be used to obtain the dispersion law [30, 26]. The DSF is calculated separately for transverse (T) and longitudinal (L) displacements. For longitudinal modes, it is defined by:

$$
\operatorname{DSF}_{L}(\omega, \mathbf{q})=\sum_{j=1}^{3 N}\left(\sum_{i=1}^{N} \mathbf{q}_{\mathbf{n}} \cdot \mathbf{u}_{\mathbf{i}}\left(\omega_{j}\right) e^{\mathbf{q} \cdot \mathbf{N}_{\mathbf{i}}}\right)^{2} \delta\left(\omega-\omega_{j}\right)
$$

where $\mathbf{q}_{\mathbf{n}}$ is the normalized wave vector and $\mathbf{N}_{\mathbf{i}}$ the position vector for the $i$ th node. As mentioned before, this expression results from the calculation of the fluctuations in the density of nodes. Writing $\rho(\mathbf{q}, t)$ the spatial Fourier components of the local density $\rho$ at time $t$, and $\rho_{\mathbf{q}}$ its initial value, we have

$$
\begin{aligned}
D_{S F_{L}}(\boldsymbol{\omega}, \mathbf{q}) & \propto \int \exp (-i \omega t)\left\langle\left(\rho(\mathbf{q}, t)-\rho_{\mathbf{q}}\right) \cdot\left(\rho(-\mathbf{q}, t)-\rho_{-\mathbf{q}}\right)\right\rangle d t \\
& \approx \int \exp (-i \omega t) \sum_{i j}\left(\mathbf{q} \cdot \mathbf{u}_{\mathbf{i}}\right)\left(\mathbf{q} \cdot \mathbf{u}_{\mathbf{j}}\right) \exp \left(i \mathbf{q} \cdot\left(\mathbf{N}_{\mathbf{i}}-\mathbf{N}_{\mathbf{j}}\right)\right) d t
\end{aligned}
$$

Indeed,

$$
\begin{aligned}
\rho(\mathbf{q}, t)-\rho_{\mathbf{q}} & =\int \rho(\mathbf{r}, t) \exp (i \mathbf{q} \cdot \mathbf{r}) d \mathbf{r}-\int \rho(\mathbf{R}, 0) \exp (i \mathbf{q} \cdot \mathbf{R}) d \mathbf{R} \\
& =\sum_{i=1}^{N} \exp \left(i \mathbf{q} \cdot\left(\mathbf{N}_{\mathbf{i}}+\mathbf{u}_{\mathbf{i}}(t)\right)\right)-\exp \left(i \mathbf{q} \cdot \mathbf{N}_{\mathbf{i}}\right) \approx \sum_{i=1}^{N} i \mathbf{q} \cdot \mathbf{u}_{\mathbf{i}}(t) \exp \left(i \mathbf{q} \cdot \mathbf{N}_{\mathbf{i}}\right)
\end{aligned}
$$

where $\mathbf{u}_{\mathbf{i}}(t)=\mathbf{r}_{\mathbf{i}}(t)-\mathbf{R}_{\mathbf{i}}$ is the displacement supported by the node $i$ in a random excitation, that is next decomposed on the eigenmodes with frequencies $\omega_{j}[31] . D_{S F_{L}}(\omega, \mathbf{q})$ thus corresponds to the Fourier transform of the longitudinal components of the waves with frequency $\omega$. Its transverse counterpart is

$$
\operatorname{DSF}_{T}(\omega, \mathbf{q})=\sum_{j=1}^{3 N}\left(\sum_{i=1}^{N} \mathbf{q}_{\mathbf{n}} \wedge \mathbf{u}_{\mathbf{i}}\left(\omega_{j}\right) e^{\mathbf{q} \cdot \mathbf{N}_{\mathbf{i}}}\right)^{2} \delta\left(\omega-\omega_{j}\right)
$$

The KPM is used once again to calculate the DSFs without exact diagonalization. With this method, DSFs can directly be computed as:

$$
\begin{aligned}
& \left.\operatorname{DSF}_{L}(\boldsymbol{\omega}, \mathbf{q})\right)=\sum_{b=0}^{B} \mu_{b} v_{b}^{L}(\mathbf{q}) T_{b}(\varepsilon) \\
& \operatorname{DSF}_{T}(\boldsymbol{\omega}, \mathbf{q})=\sum_{b=0}^{B} \mu_{b} v_{b}^{T}(\mathbf{q}) T_{b}(\varepsilon)
\end{aligned}
$$

with $\mu_{b}$ and $\varepsilon$ as previously defined, and:

$$
\begin{gathered}
v_{b}^{L}(\mathbf{q})=\overline{\frac{1}{N}\left(\sum_{i=1}^{N} \mathbf{q}_{\mathbf{n}} \cdot \mathbf{u}_{\mathbf{i}}^{0} e^{\mathbf{q} \cdot \mathbf{N}_{\mathbf{i}}}\right)^{2}\left(\sum_{i=1}^{N} \mathbf{q}_{\mathbf{n}} \cdot \mathbf{u}_{\mathbf{i}}^{b} e^{\mathbf{q} \cdot \mathbf{N}_{\mathbf{i}}}\right)^{2}} \\
v_{b}^{T}(\mathbf{q})=\overline{\frac{1}{N}\left(\sum_{i=1}^{N} \mathbf{q}_{\mathbf{n}} \times \mathbf{u}_{\mathbf{i}}^{\mathbf{0}} e^{\mathbf{q} \cdot \mathbf{N}_{\mathbf{i}}}\right)^{2} \cdot\left(\sum_{i=1}^{N} \mathbf{q}_{\mathbf{n}} \times \mathbf{u}_{\mathbf{i}}^{\mathbf{b}} e^{\left.\mathbf{q} \cdot \mathbf{N}_{\mathbf{i}}\right)^{2}},\right.}
\end{gathered}
$$

\subsection{Voronoi Decomposition}

The decomposition of the displacement on longitudinal and transverse components compared to the wavevector is not straightforward for non-crystalline samples such as the Octagonal quasi-periodic tiling [32]. The displacement decomposition used herein is based 
on the Voronoi cells volume variation and was already applied to amorphous glassy samples in [19]. The Voronoi cell built around a node is the area containing the points closer to this node than every others. The Transverse displacements will be the displacements not modifying the Voronoi cells' volume and the Longitudinal are the displacements doing so. As in [19], we use the A matrix describing the relative volume variation of the voronoi cell centered on node $i$, due to the displacement $d_{j}$ of node $j$ (translation $u_{x}, u_{y}$ or rotation $\theta$ ):

$$
A_{i, j}=\frac{1}{V_{i}} \frac{\partial V_{i}}{\partial d_{j}}
$$

This matrix can be easily obtained from the mesh of beam elements, the detailed method being described in [19]. With this definition of $\mathbf{A}$, we obtain the longitudinal and transverse components by:

$$
\begin{gathered}
\mathbf{d}_{\eta}=\mathbf{P}_{\eta} \mathbf{d} ; \eta=L, T \\
\mathbf{P}_{\mathbf{L}}=\mathbf{A}^{t}\left(\mathbf{A A}^{t}\right)^{-1} \mathbf{A} \\
\mathbf{P}_{\mathbf{T}}=\mathbf{I}-\mathbf{A}^{t}\left(\mathbf{A} \mathbf{A}^{t}\right)^{-1} \mathbf{A}
\end{gathered}
$$

This new decomposition is then injected in the VDOS and the DSF calculation. The contribution to the VDOS of the Tchebychev moment (Eq. 177) for example, becomes:

$$
\mu_{b}^{\eta}=\overline{\left\langle\mathbf{d}_{0}^{r}\left|\mathbf{P}_{\eta}\right| \mathbf{d}_{b}^{r}\right\rangle}
$$

with $\eta=L$ or $\eta=T$ for its respective longitudinal and transverse contributions to the VDOS.

\section{Role of bending in periodic beam lattice}

As a first example, the vibration of a periodic beam lattice analyzed. The effect of the bending stiffness that is the only design parameter to be adjusted once the geometry of the lattice is fixed, is investigated. The ratio between the tensile stiffness and the bending stiffness is varied in order to observe the influence of the latter on the vibrational response.

145 The idea behind such a study is that increasing local bending stiffness should enhance the energy separation between local rotational and compressional modes, and thus enlarges possible hybridization gaps (see e.g. [33]) meaning they result from the interaction between modes of different nature.

\subsection{Analytical solution for a periodic lattice}

A infinite periodic lattice with a simple squared elementary pattern is considered (see Figure 3). As for wave propagation [34], the periodic structure of this lattice allows the use of periodic boundary conditions to reduce the analysis to one elementary cell. Using those conditions and choosing the solutions of Equation (3) as:

$$
\mathbf{d}=\mathbf{a}_{\mathbf{d}} \exp (i(\omega t+\mathbf{q} \cdot \mathbf{r})),
$$

equation 3 is solved analytically. This calculation is detailled in Appendix $\mathrm{A}$. The values $[\omega, \mathbf{q}]$ that verify $\operatorname{det}\left(\left([K]-\omega^{2}[M]\right)\right)=0$ are calculated using the MAPLE software.

Figures 4 a-b-c show the resulting analytical solution of the dispersion law (DSF) for three ratios $\frac{K_{v}}{K_{u}}$, namely $0.01,0.5$ and 2 . For graphical representation, the dimensionless frequency $\omega_{\text {adim }}$ defined as $\omega_{\text {adim }}=\omega \sqrt{\frac{m}{K_{u}}}$, is used.

For the three cases analyzed herein, three surfaces $\left(\omega_{\text {adim }}\right.$ as a function of $\left.q_{x}, q_{y}\right)$ are plotted: two acoustic solutions (in phase vibrations of the nodes: blue and red) and one optical (out of phase vibrations of the nodes: green). For $\frac{K_{v}}{K_{u}}=0.01$ and $\frac{K_{v}}{K_{u}}=0.5$, the green and blue surfaces merge at $\left(\frac{\pi}{L}, \frac{\pi}{L}\right)$ whereas for $\frac{K_{v}}{K_{u}}=2$ the two surfaces are disconnected. Note that for $\left(\frac{\pi}{L}, \frac{\pi}{L}\right)$, the vibration modes correspond to uncoupled vibrations of the three 
nodal degrees of freedom. This creates a frequency range in which no mode exists. This is called a band gap. It can be shown that the analytical solution used above degenerates for $\left(q_{x}, q_{y}\right)$ reaching $\left(\frac{\pi}{L}, \frac{\pi}{L}\right)$ giving a double solution and a single one:

$$
\left\{\sqrt{\frac{210\left(K_{v}+K_{u}\right)}{43 m}}, \sqrt{\frac{10 K_{v}}{m}}\right\}
$$

Using the expression of those solutions, the size of the band gap depending on $\frac{K_{v}}{K_{u}}$ is :

$$
\Delta \omega_{\text {adim }}=\sqrt{\frac{m}{K u}}\left(\sqrt{\frac{10 K_{v}}{m}}-\sqrt{\frac{210\left(K_{v}+K_{u}\right)}{43 m}}\right)^{+}
$$

155

where the + superscript indicates the positive part of a real number. It is obtained that below a given ratio $\frac{K_{v}}{K_{u}}$, no band gap is obtained whereas above a critical value of 0.9545 , the size of the band gap increases as $\sqrt{\frac{K_{v}}{K_{u}}}$ as illustrated in Figure 5

As shown in Figures $4-\mathrm{b}$ and 4 - $\mathrm{c}$, the high-frequency modes in the periodic beam lattice are mainly transverse. Increasing $K_{v}$ independently of $K_{u}$ thus raises mainly the frequency of optical transverse modes (upper band in the dispersion relation), creating a band gap. Note that $\frac{K v}{K u}$ ratio higher than 0.05 (slenderness approximately lower than 5) can't be geometrically obtained for square section beams while respecting the slenderness requirements for the Euler-Bernoulli beam model, thus making impossible to obtain band gaps for these specific stiffness values. It may however be possible to create a band gap by adjusting the mass distribution of the beams or just with a different periodic structure. The goal of this article is indeed to show evidence of tendencies that could be even amplified with a more accurate description of the interactions beyond the Euler-Bernouilli approximation, and to adapt new tools to the study of the vibrations in non-periodic beam structures. The three typical ratios of $K_{v} / K_{u}=0.01,0.5$ and 2 will thus be kept all-along the article for comparison purposes.

\subsection{Numerical calculations}

To further analyze the vibrational behaviour of the square lattice, the PR and VDOS have been computed from a numerical solution obtained for a $5 \times 5$ to $50 \times 50$ cells lattice as presented in Figure 3 . For the $50 \times 50$ lattice, the system has $N=2601$ nodes for $3 N(=$

175 7803) DFs, including the boundary. Periodic boundary conditions are applied along the boundary of the analyzed domain. For these simulations, the parameters used were $L=$ $0.01 \mathrm{~m}, K_{u}=3.5 \times 10^{5} \mathrm{~kg} . \mathrm{s}^{-2}, \rho=1000 \mathrm{~kg} . \mathrm{m}^{-3}, E=1.4 \times 10^{9} \mathrm{~Pa}, K_{v}=2 K_{u}, K_{v}=0.5 K_{u}$ and $K_{v}=0.01 K_{u}$.

For the three values of $\frac{K_{v}}{K_{u}}$, the VDOS and PR are computed following the methodology detailed above. The results are plotted in Figure 6 to check the influence of finite size effects, and in Figures 7, 8 and 9 for different values of $K_{v} / K_{u}$. In the latter, i.e. for $\frac{K_{v}}{K_{u}}=2$, the creation of the band gap is clearly observed in the VDOS and in the PR. Indeed, for around $\omega_{\text {adim }} \approx 4$ that corresponds to our analytical value of $\omega_{\text {adim }}$ in the band gap, the VDOS vanishes and there is no defined value for the PR as no vibration mode exists for $\omega_{\text {adim }}$ within the band gap. Conversely, for $\frac{K_{v}}{K_{u}}=0.5$ and $\frac{K_{v}}{K_{u}}=0.01$, the VDOS does not vanish and there is no zone with undefined PR. However, for all three cases, the fluctuations of the VDOS are in close relation with the analytically obtained shape of the surfaces giving the three different roots $\omega_{\text {adim }}$ as plotted in Figures $4-a-b-c$. Concerning the PR, most of the modes have a PR around 0.6 due to the fact that it is the PR of a spatial cosine to which all the nodes participate. This example shows how the VDOS and the PR can be used to interpret the vibrational behavior of the material and to detect band gaps. As expected, it shows as well, that the additional flexural stiffness in the beam lattice model induces a new kind of high frequency optical modes, with mainly transverse character and with the related aperture of a band gap increasing with $K_{v}$ in the vibrational response. 


\section{Quasi-periodic beam lattice}

The methodology is now applied to analyze the behaviour of a quasi-periodic beam lattice. The complexity of quasi-periodic structures does not allow the calculation of analytical solutions as was done previously. Only numerical results are obtained. All the calculations are run on the $4^{\text {th }}$ order periodic approximant of a Octogonal quasi-periodic tiling

200 shown on Figure 2. The details for the generation of this quasi-periodic approximant are given in [35]. The corresponding quasi-periodic tiling is the octogonal Amman-Beenker's tiling initially proposed in [36]. The system has $N=8257$ nodes and $3 N(=24771)$ DFs. As previously, periodic boundary conditions are prescribed. The parameters of the model are the same as previously: $L=0.01 \mathrm{~m}, K_{u}=3.5 \times 10^{5} \mathrm{~kg} . \mathrm{s}^{-2}, \rho=1000 \mathrm{~kg} . \mathrm{m}^{-3}$, $E=1.4 \times 10^{9} \mathrm{~Pa}$. To understand the influence of the flexural component of the beam stiffness, the same three values of $\frac{K_{v}}{K_{u}}$ are still considered: 0.01, 0.5 and 2.

For the three values of $\frac{K_{v}}{K_{u}}$, the VDOS and PR are shown in Figures 10 , 11 and 12 . The VDOS of the quasi-periodic tiling considered in this section, share some similarities with the ones computed for the periodic square lattice: for the three values of $\frac{K_{v}}{K_{u}}$, there is first the VDOS increases again which gives an indication concerning the existence of optical branches (high frequency out-of-phase vibrations of the nodes with low group velocity). Concerning the PR, whereas for the square lattice there is no clear trend in the evolution of the PR, the quasi-periodic tiling analyzed herein behaves differently. It is observed that the PR follows fluctuations that seem (quite surprisingly) opposite to those of the VDOS for $\frac{K_{v}}{K_{u}}=0.5$ and $\frac{K_{v}}{K_{u}}=2$, but similar for $\frac{K_{v}}{K_{u}}=0.01$ except around $\omega_{\text {adim }}=0.6$. In these cases, a drop of the PR relates to a peak in the VDOS. Consequently, in the frequency ranges with a high density of modes, the latters have the tendency to show localized patterns. Conversely, in the frequency ranges with a low density of modes, the latters show diffuse ins: especially in the high frequency regime, quasi-crystalline structures do not give rise to localized modes, but more surprisingly to a $P R \approx 0.6$ close to that of extended plane waves. It can finally be noticed a higher concentration of modes around certain frequencies, notably in the high frequency range, which results in waviness of the VDOS. In order to apprehend how the quasi-periodic tiling vibrates, it can be interesting to look at several modes on specific domain of the frequency range. Several modes are plotted for $\frac{K_{v}}{K_{u}}=0.01$ (see Supplementary Material for all the modes). Figures 13 and 14 show highly structured modes where the vibration is localized on a star shaped structure as in Figure 17. These modes, localized on stars, are involved repeatedly in the two regions where the PR decreases. These modes are localized on specific patterns of the lattice. Figure 16 shows 230 that, in the second decaying region of the PR, another kind of localized modes appears as well: they involve a thin but extended crowned of vibrations. This kind of localization is very surprising and specific of quasi-crystals: it shows large scale vibrations in the high frequency regime. Finally, a complete set of structures with various sizes is excited along the modes and disordered patterns can also be found as in Figure 15 in a frenquency range where the PR is higher.

In order to test the hypothesis of isolated vibrations of specific patterns in the low frequency regime, that would be decorrelated from the overall environment, the star structure has been isolated and its own vibrational modes have been studied with fixed boundary conditions. In Figure 18 and 19 the VDOS of the star is compared to the frequency dependence

240 of the PR of the $4^{\text {th }}$ approximant for two ratios $\frac{K_{v}}{K_{u}}$. In both cases, the frequency for which this structure plays a predominant role in the vibrations of the whole structure - as characterized by the marked local minimum in the PR - corresponds to the frequency range of a peak of the VDOS - especially of volume preserving modes VDOST - for the isolated star. This might be the cause of the waviness of the VDOS: the vibrational modes concentrate around the frequencies that excite particular sub-structures. This response again is specific of quasi-crystals, since it shows evidences of the resonant vibrations of isolated structures, even in the low-frequency regime (isolated soft resonators). In this low-frequency range, 
extended vibrations are expected in crystals. These specific resonant vibrations however do not correlate with a band gap.

250 For $\frac{K_{v}}{K_{u}}=2$, an additional gap is observed in the VDOS Figure 12 meaning that increasing the bending stiffness strongly impacts the vibrational behavior of the quasi-periodic lattice, namely by creating additional band gaps. The evolution of those gaps is also observed thanks to the DSF shown in Figures 20 and 21. The maxima of DSF intensity allow a quick visualization of the dispersion law, but in the case of a quasi-crystal, a given frequency $\omega$ does not correspond to a single wave-vector $q$. Those figures exhibit classical dispersion relations for quasi-periodic structure as it can be found in [37, 1, 5] for 1D or 2D quasi-periodic lattices. We can observe for example pseudo Bernoulli zones overlapping and repeating at quasi-periodic periods. But an additional gap is visible in Fig. 21 for $K_{v} / K_{u}=2$, as can be seen from the extinction of the DSF close to $\omega \approx 4$. This large gap is 260 visible in the dispersion law shown in Figure 21 especially for longitudinal modes.

\section{Conclusion}

The vibrational properties of periodic and quasi-periodic beam lattices were studied in this paper, as a function of the ratio between bending and tensile stiffness of the beams. The Vibrational Density of States, Participation Ratio and Dynamical Structure Factors for

265 longitudinal as well as for transverse waves have been investigated for different ratios of bending over tensile stiffness. This ratio appears to be a driving parameter for large band gaps to occur and is related to an enhanced separation between in-phase tensile modes and out-of-phase bending vibrations. It is interesting to note that, contrary to the periodic lattice which has anisotropic effective behavior for elasticity and for wave propagation, the Quasi-periodic beam structure, that has a higher level of material symmetry, is an apparent isotropic metamaterial. This was already discussed in [38, 39]. The creation of band gaps in the numerical simulations of the Quasi-periodi structure is thus the manifestation of a structural intrinsic property scaling with $K_{v} / K_{u}$. Then even if the band gaps are only reached here with unrealistic geometries in terms of beam slenderness assuming square

275 cross section, it might be possible to obtain band gaps by adjusting other parameters of the structure (mass distribution, viscosity, etc ) chosen with the same goal of frequency separation between in-phase tensile and out-of-phase bending modes. Finally, the position of the gap scales with $\sqrt{K_{u} / m}=\sqrt{E / \rho L^{2}}$ that depends on the size as well as on the material properties of the elementary beams.

Some low-frequency modes of the quasi-periodic lattice seems to be controlled by substructures of the lattice: indeed, the frequencies of these modes in the quasi-crystal correspond exactly to the frequency of the isolated local structure that appears repeatedly in the vibration modes. Conversely, high-frequency localized modes in the quasi-crystal involve large scale linear structures, suggesting the possibility to isolate large-scale and highly

285 symmetric connected paths in the quasy-cristal. These resonant isolated structures are not sufficient to induce additional band gaps. A way to select the vibration modes by the type of structure they excite, eventually by reinfocing locally specific structures with additional masses, could allow revealing new patterns in PR, VDOS or DSF. For instance, it could be interesting to study more accurately and systematically the non-trivial hierarchy of sizes it could have additional consequences as well on the transportation of wave packets with different wavelengths, and thus on acoustic signal processing.

\section{Acknowledgment}

Discussions with Remy Mosseri and Marc de Boissieu greatly helped our understanding of the quasi-periodic lattices. Discussion with Yaroslav Beltukov is highly acknowledged for the use of the KPM method. The supports of CNRS through METAMORPH 
PEPS project and ANR through grant ANR-16-CE30-0007-01 are also gratefully acknowledged. 
Figures

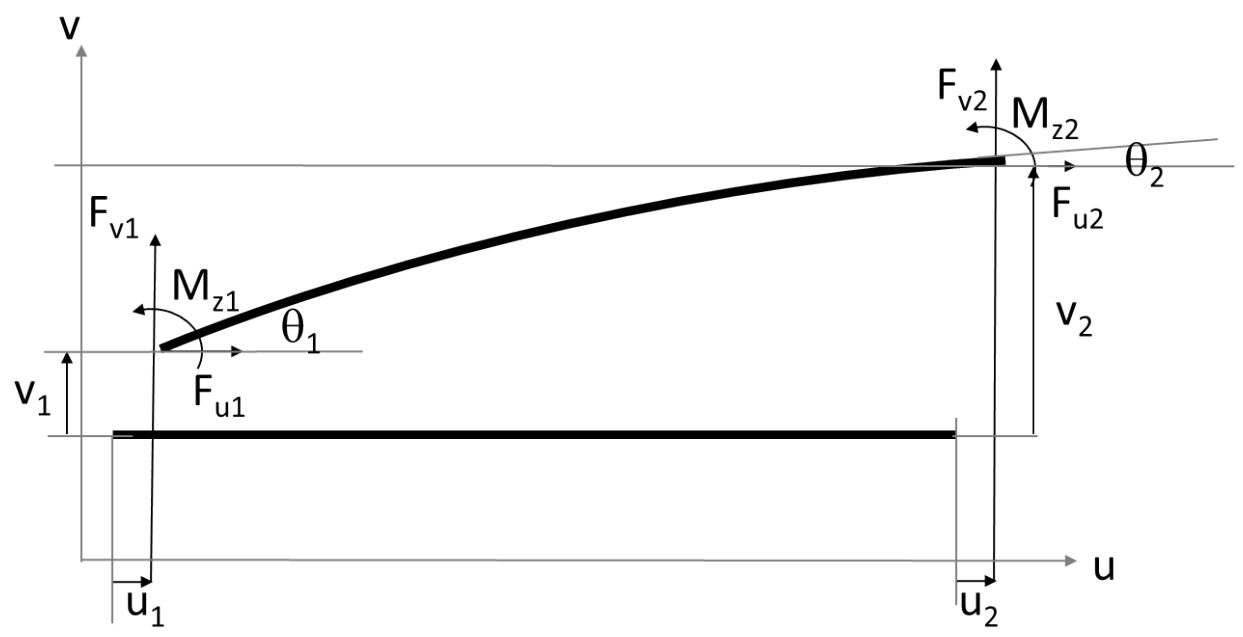

Figure 1: Representation of an isolated beam, and of its degrees of freedom. 


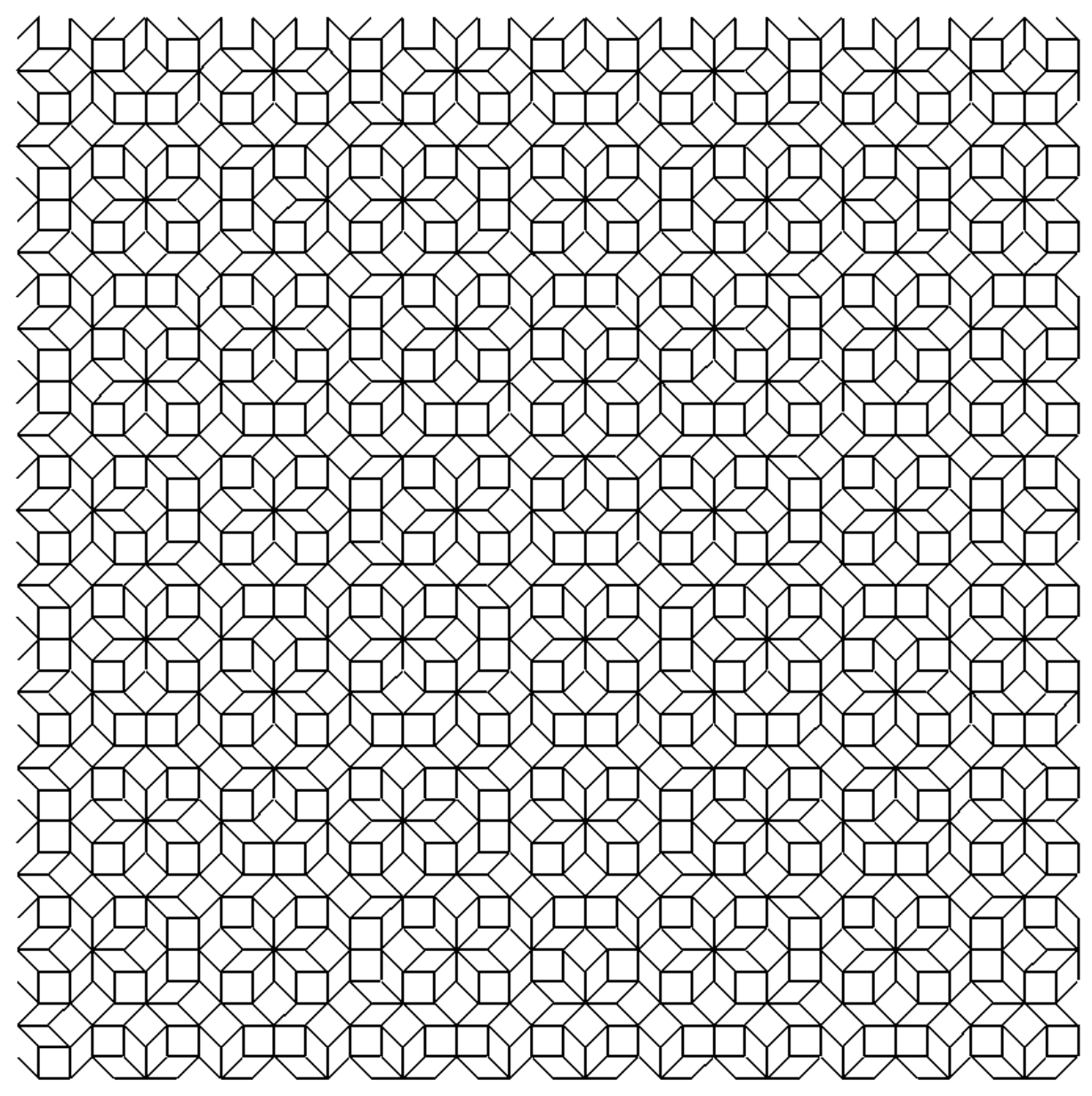

Figure 2: 4th approximant of Octagonal quasi-periodic tiling beam structure. 


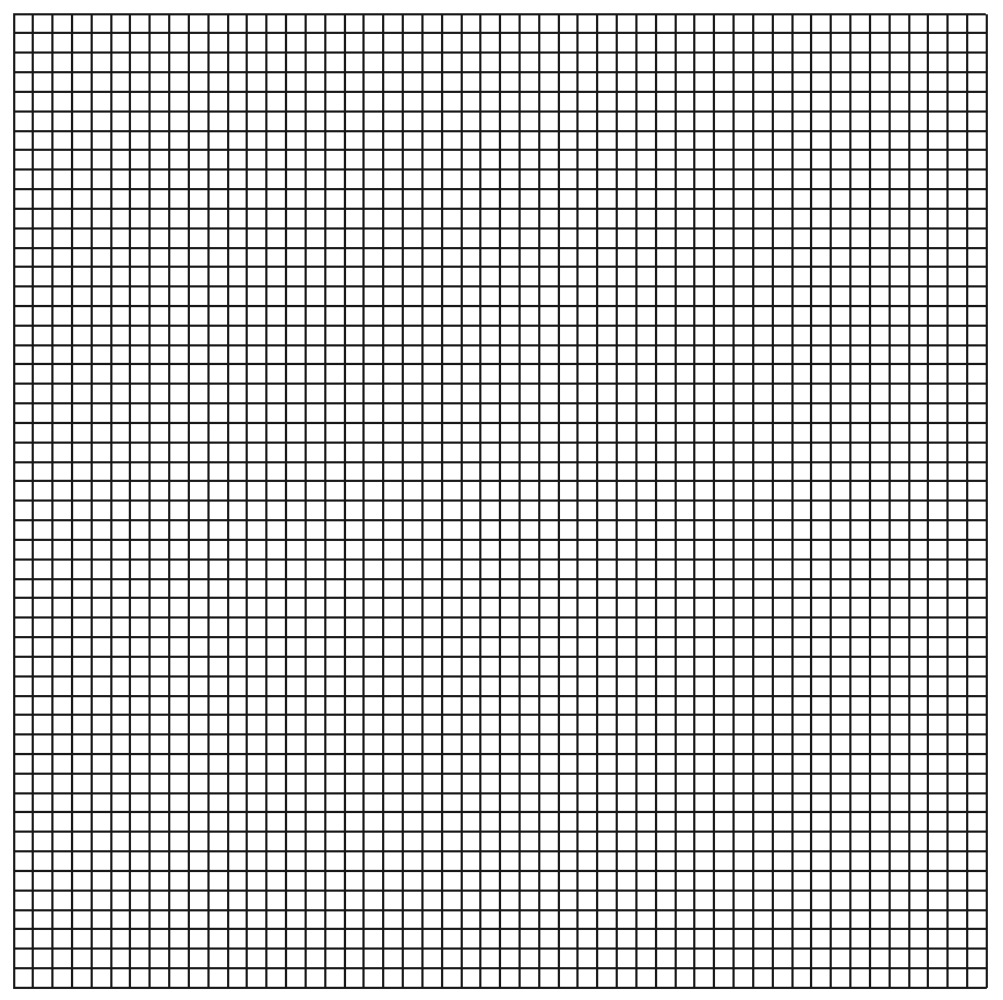

Figure 3: $50 \times 50$ square beam structure. 


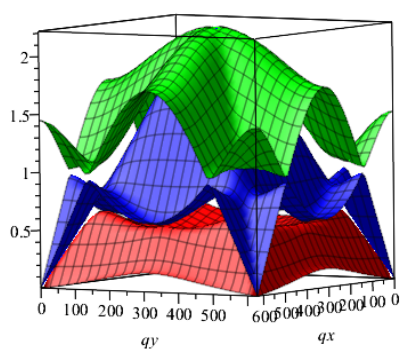

(a)



(b)

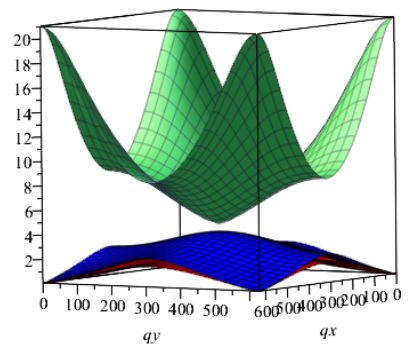

(c)

Figure 4: Analytical dispersion relation $\omega_{a d i m}\left(q_{x}, q_{y}\right)$ for an infinite Square lattice with (a) $\frac{K_{v}}{K_{u}}=0.01$ (b) $\frac{K_{v}}{K_{u}}=0.5$ (c) $\frac{K_{v}}{K_{u}}=2$. 


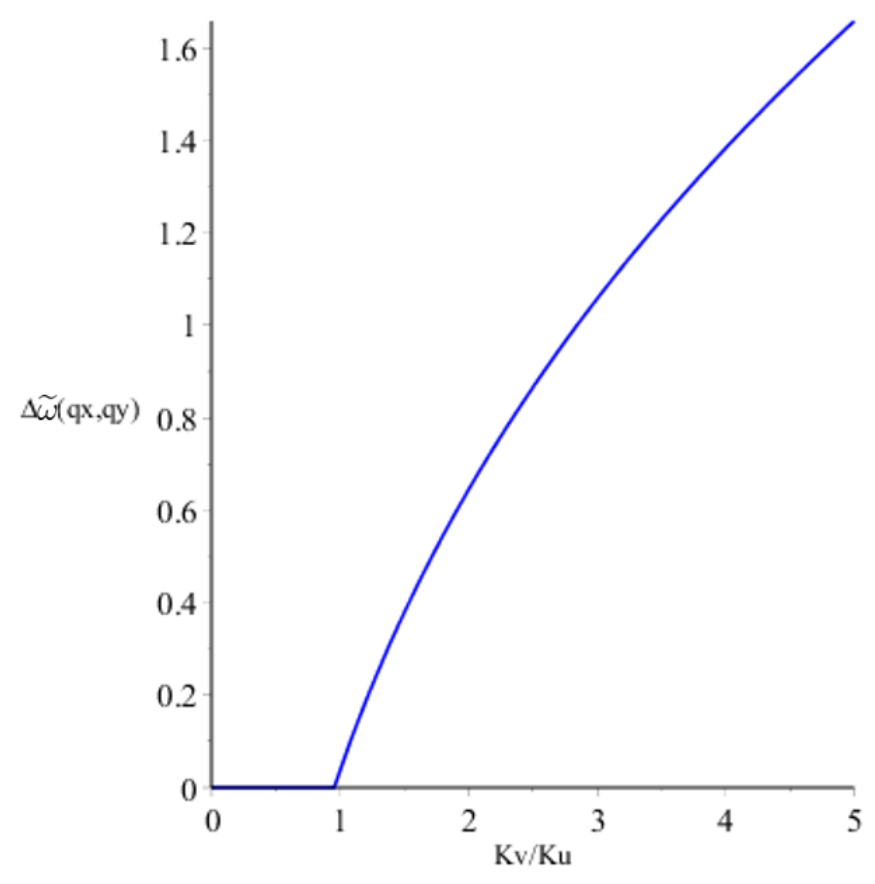

Figure 5: Analytical band gap for an infinite square lattice in function of $\frac{K_{v}}{K_{u}}$.

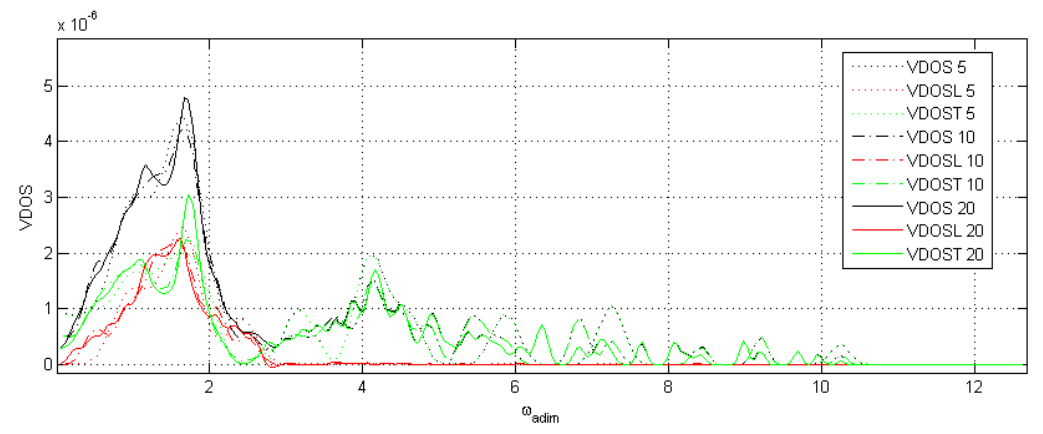

Figure 6: VDOS for the $5 \times 5,10 \times 10$ and $20 \times 20$ square lattice with $\frac{K_{v}}{K_{u}}=0.5$. VDOSL in the longitudinal VDOS and VDOST is the transverse VDOS, as discussed in the text. 




(a)

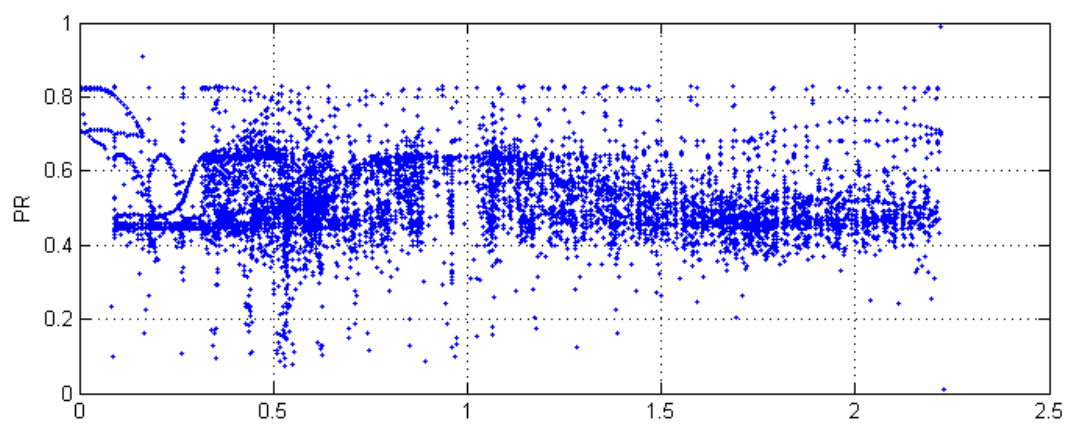

(b)

Figure 7: (a) Complete, longitudinal and transverse VDOS and (b) PR for the $50 \times 50$ square lattice with $\frac{K_{v}}{K_{u}}=0.01$. 


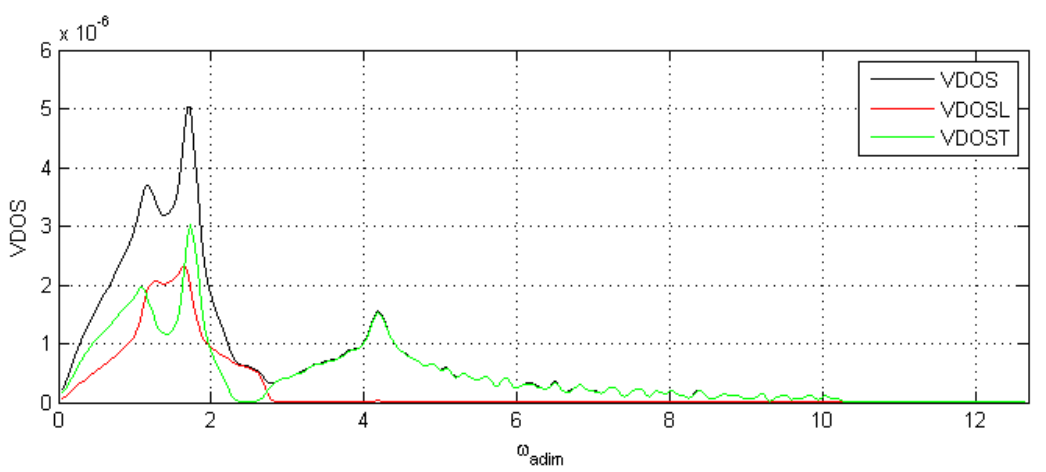

(a)

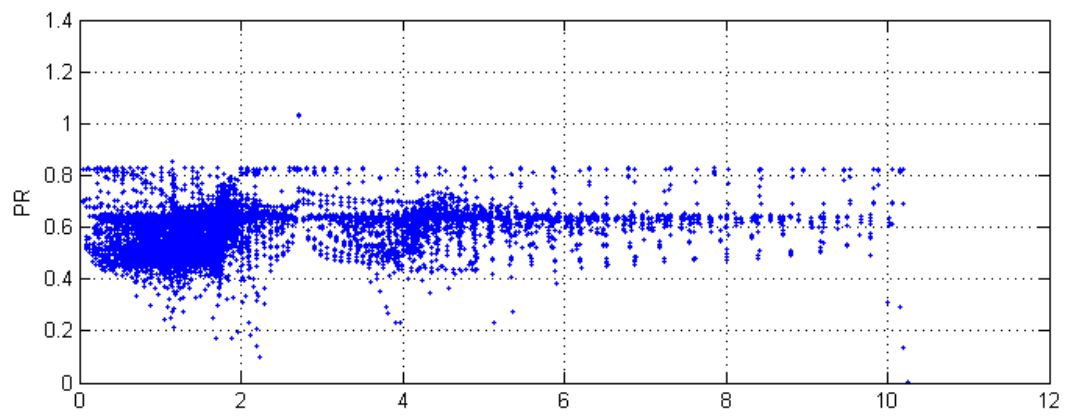

(b)

Figure 8: (a) Complete, longitudinal and transverse VDOS and (b) PR for the $50 \times 50$ square lattice with $\frac{K_{v}}{K_{u}}=0.5$. 


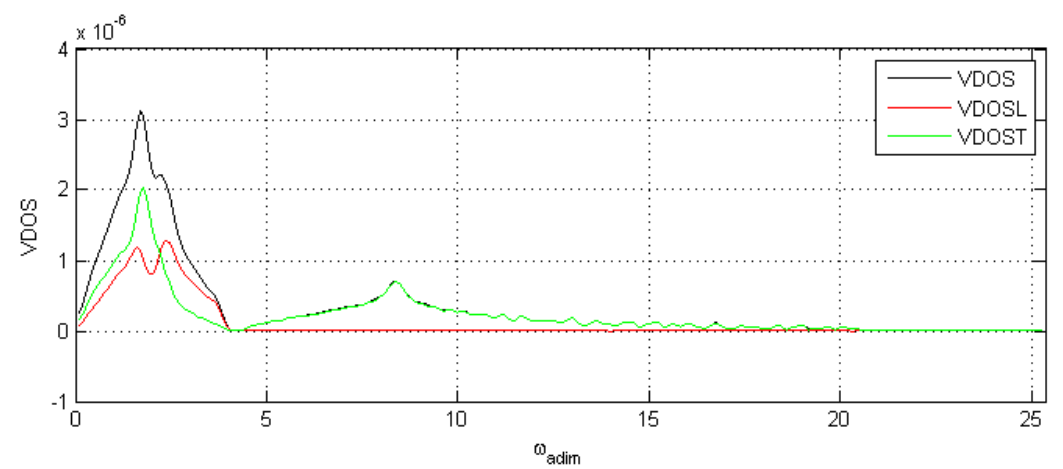

(a)

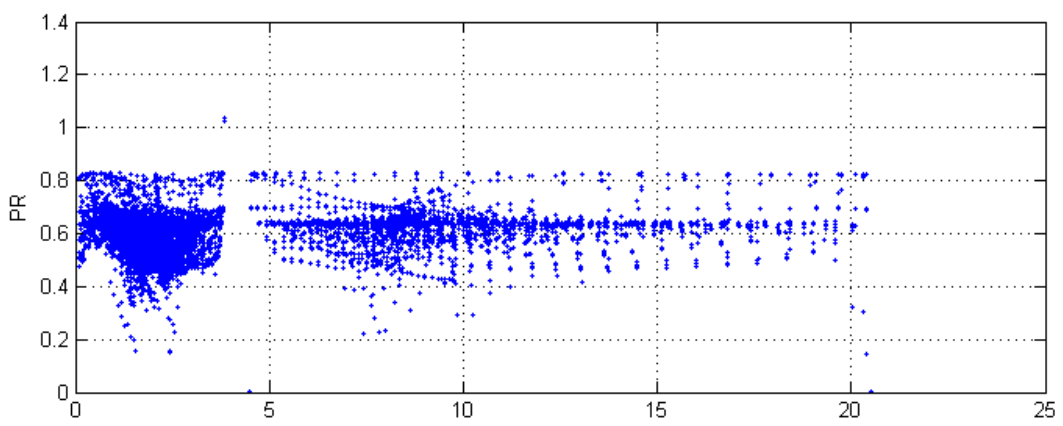

(b)

Figure 9: (a) Complete, longitudinal and transverse VDOS and (b) PR for the $50 \times 50$ square lattice with $\frac{K_{v}}{K_{u}}=2$. 


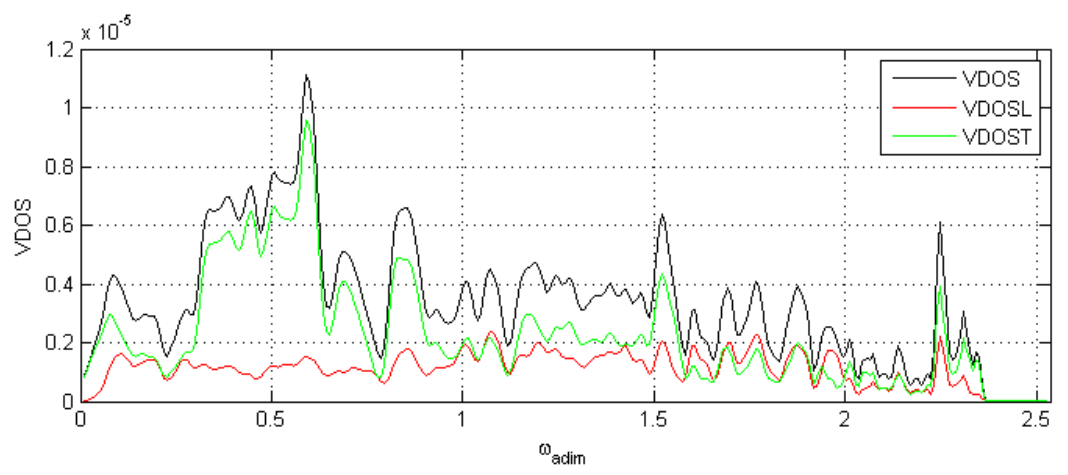

(a)

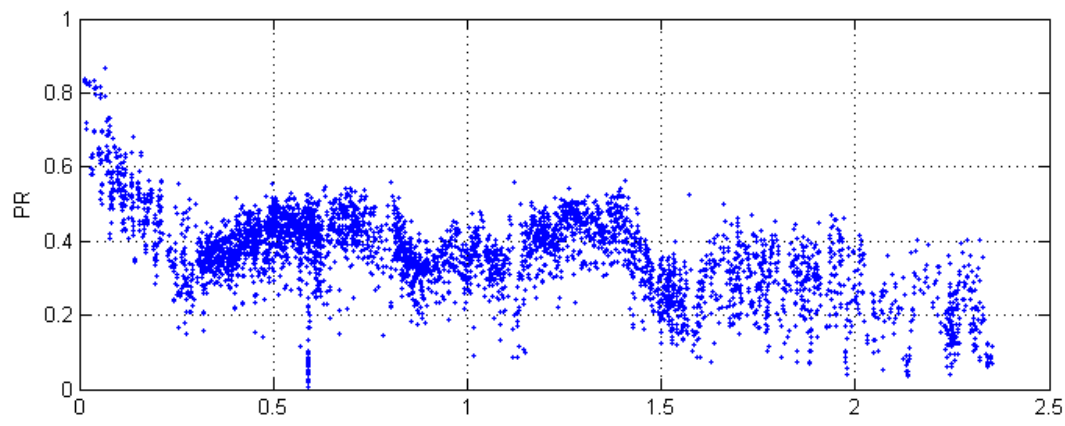

(b)

Figure 10: (a) Complete, longitudinal and transverse VDOS and (b) PR for the 4th approximant of the Octagonal Quasi-periodic tiling beam structure with $\frac{K_{v}}{K_{u}}=0.01$. 


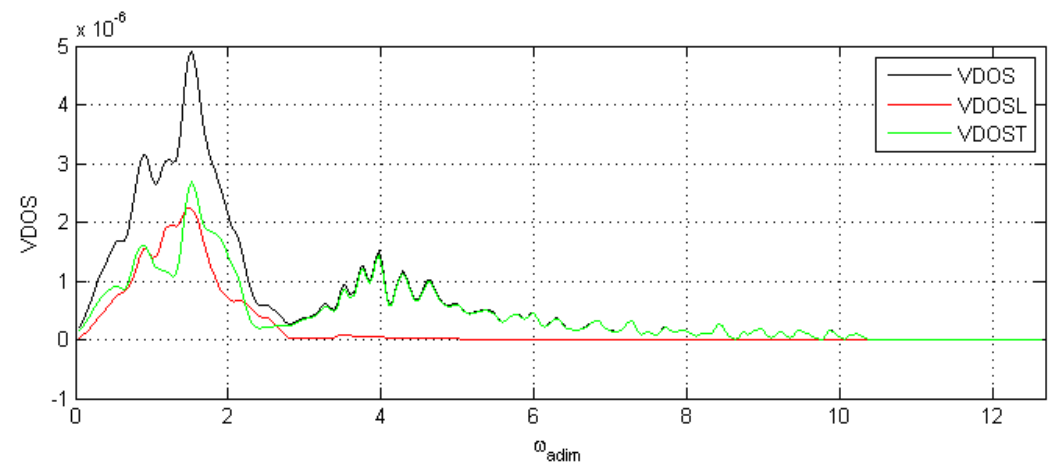

(a)

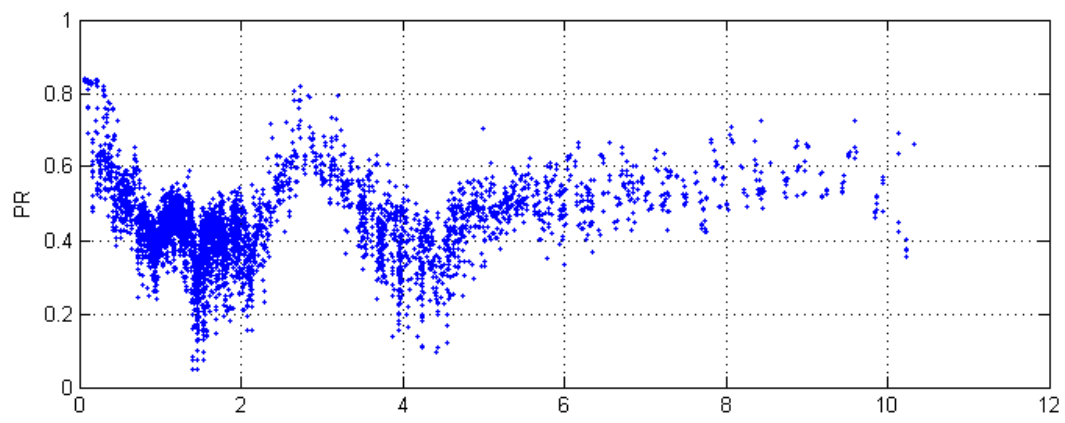

(b)

Figure 11: (a) Complete, Ingitudinal and transverse VDOS and (b) PR for the 4th approximant of the Octagonal Quasi-periodic tiling beam structure with $\frac{K_{v}}{K_{u}}=0.5$. 


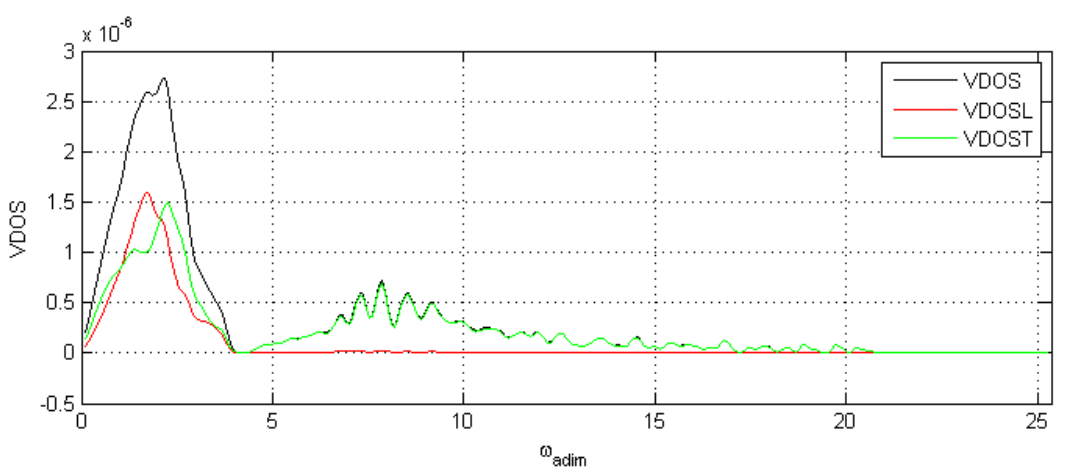

(a)

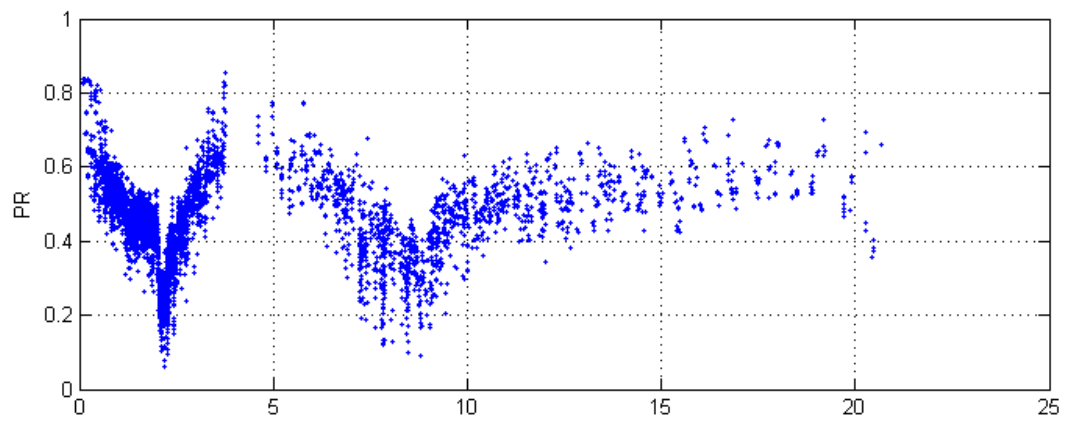

(b)

Figure 12: (a) Complete, longitudinal and transverse VDOS and (b) PR for the 4th approximant of the Octagonal Quasi-periodic tiling beam structure lattice with $\frac{K_{v}}{K_{u}}=2$. 


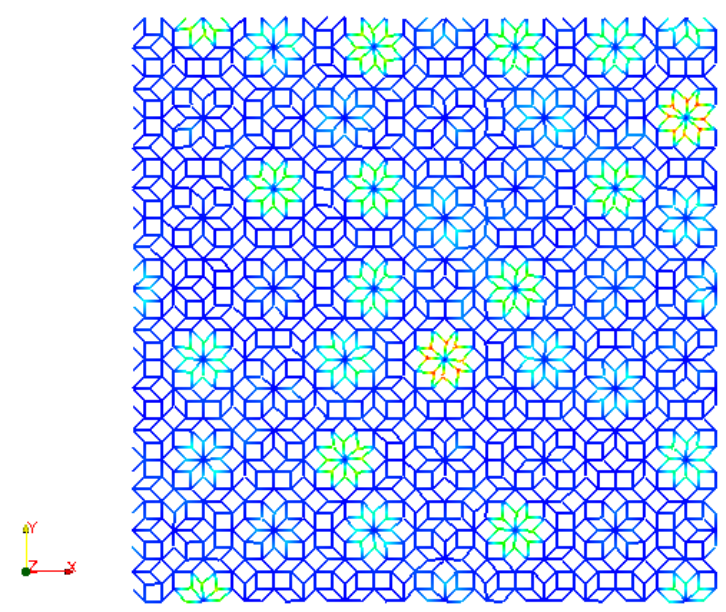

(a)

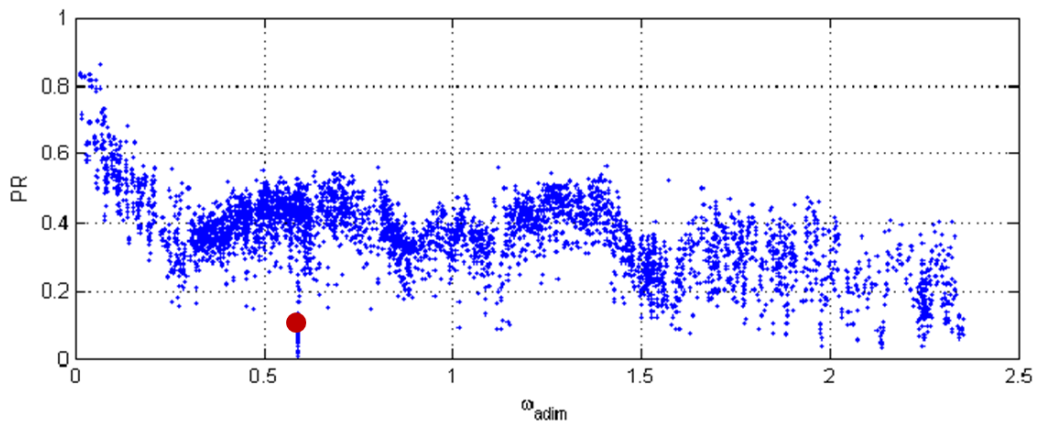

(b)

Figure 13: (a) Deformed lattice and (b) PR for the $4^{\text {th }}$ approximant of the Octagonal Quasi-periodic tiling beam structure with $\frac{K_{v}}{K_{u}}=0.01$. The full PR is in blue, and the red circle indicates the PR and the frequency of the $1387^{\text {th }}$ mode shown above. 


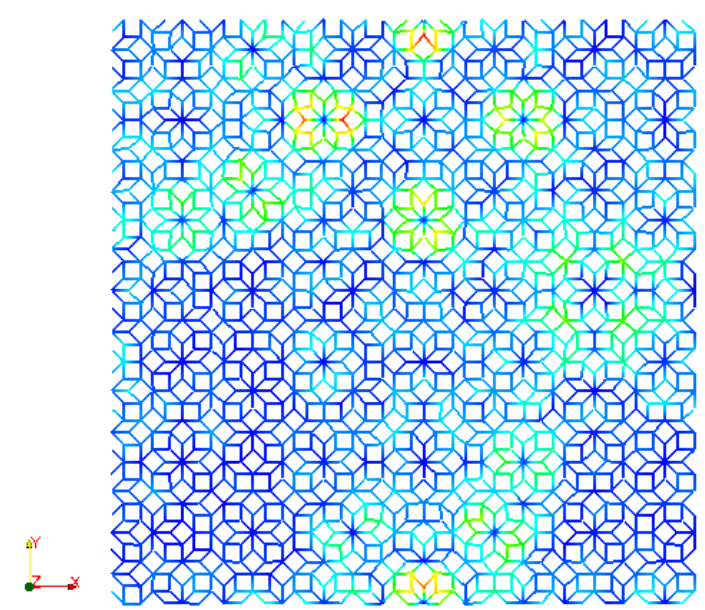

(a)

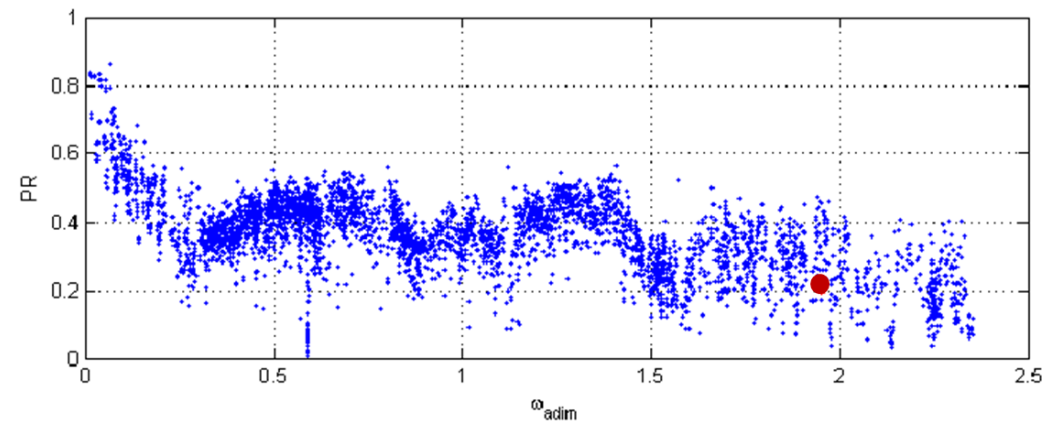

(b)

Figure 14: (a) Deformed lattice and (b) PR for the $4^{\text {th }}$ approximant of the Octagonal Quasi-periodic tiling beam structure with $\frac{K_{v}}{K_{u}}=0.01$. The full PR is in blue, and the red circle indicates the PR and the frequency of the $3836^{\text {th }}$ mode shown above. 


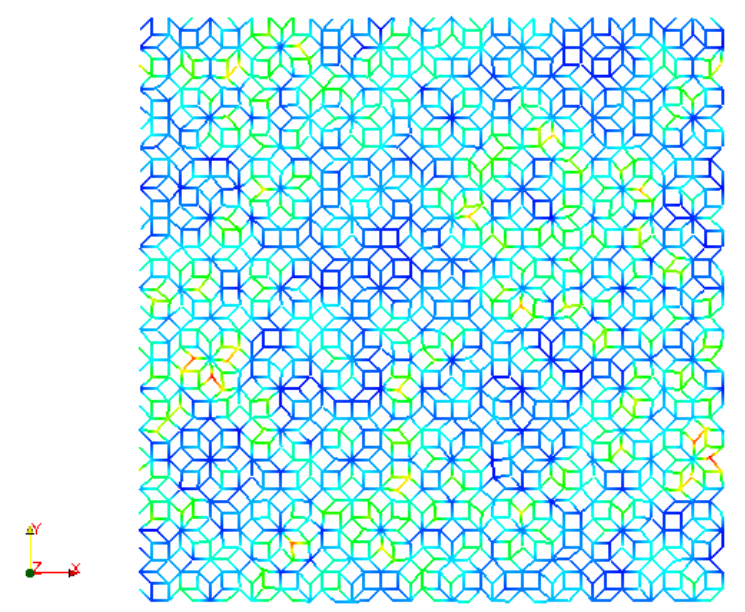

(a)

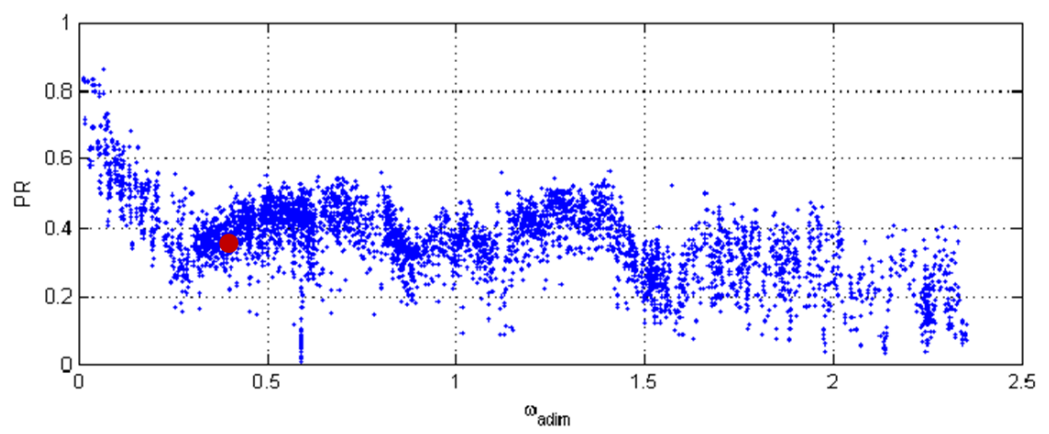

(b)

Figure 15: (a) Deformed lattice and (b) PR for the $4^{\text {th }}$ approximant of the Octagonal Quasi-periodic tiling beam structure with $\frac{K_{v}}{K_{u}}=0.01$. The full PR is in blue, and the red circle indicates the PR and the frequency of the $693^{r d}$ mode shown above. 


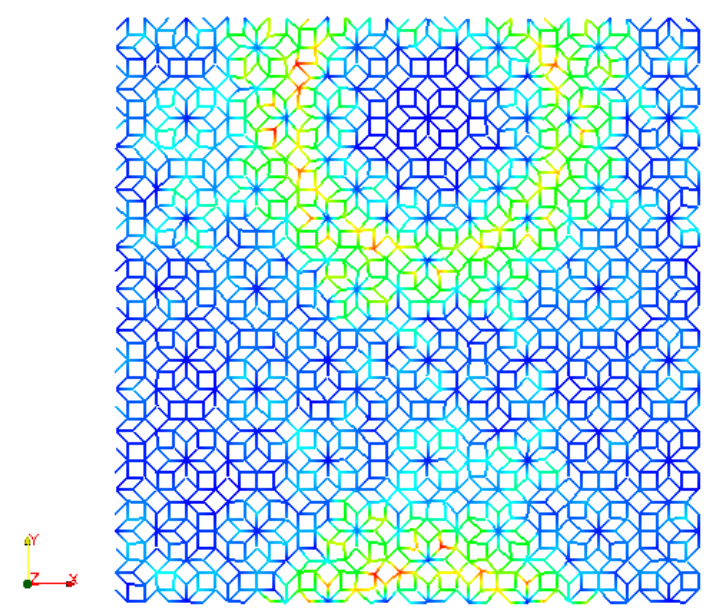

(a)

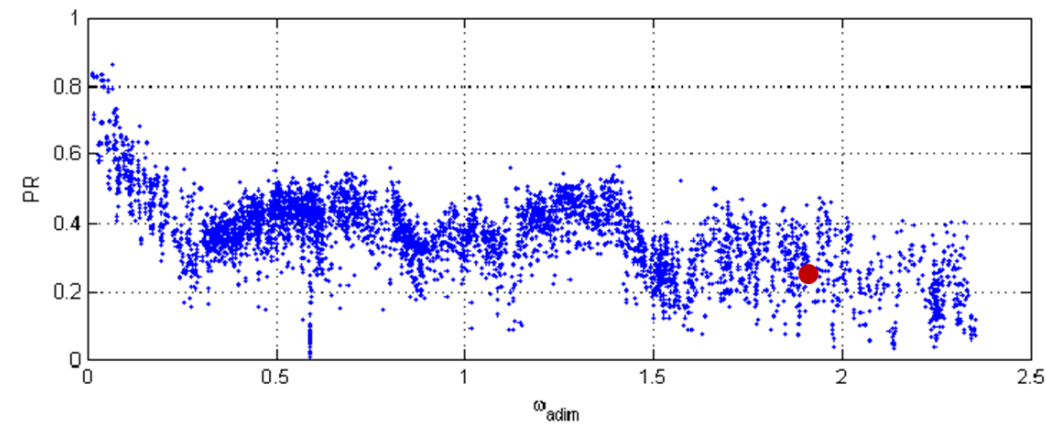

(b)

Figure 16: (a) Deformed lattice and (b) PR for the $4^{\text {th }}$ approximant of the Octagonal Quasi-periodic tiling beam structure with $\frac{K_{v}}{K_{u}}=0.01$. The full PR is in blue, and the red circle indicates the PR and the frequency of the $3801^{r s t}$ mode shown above. 


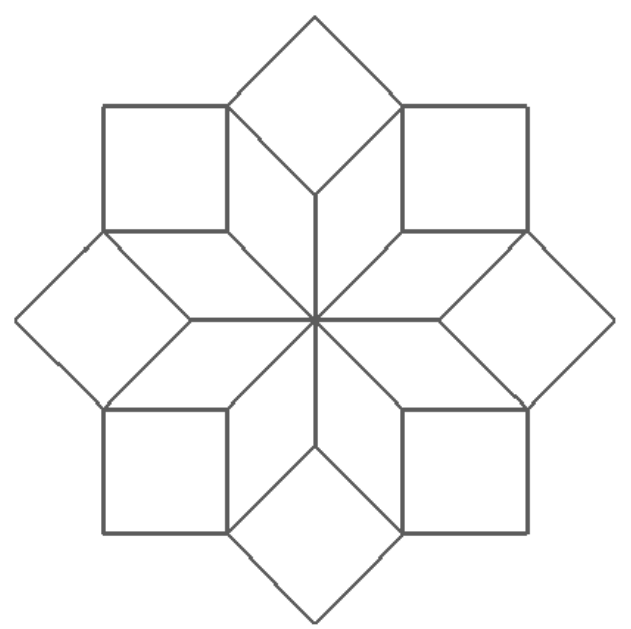

Figure 17: Star structure.

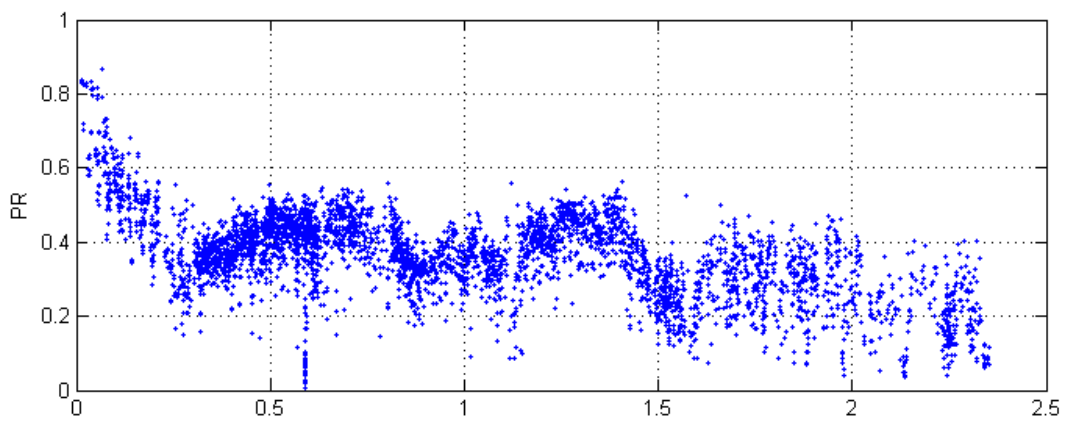

(a)

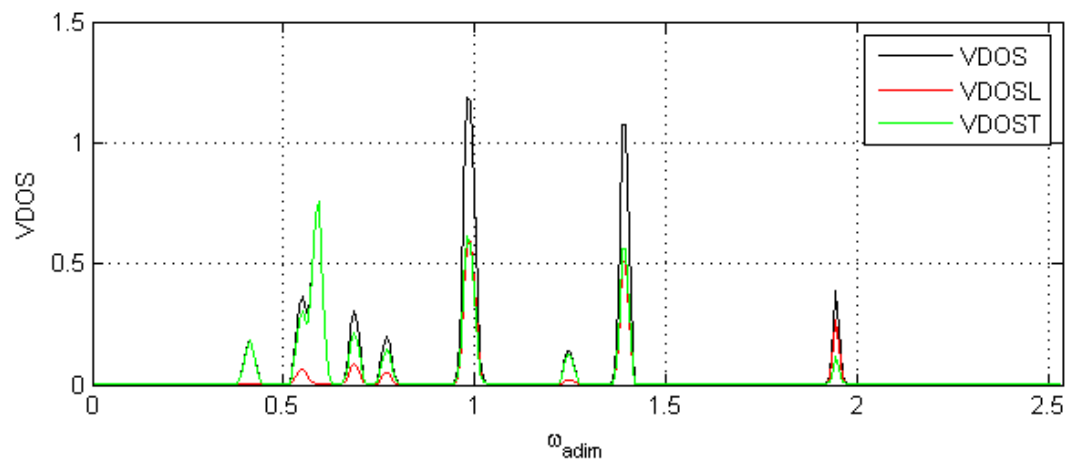

(b)

Figure 18: (a) PR and (b) complete, longitudinal and transverse VDOS of the isolated star, for the $4^{\text {th }}$ approximant of the Octagonal Quasi-periodic tiling beam structure with $\frac{K_{v}}{K_{u}}=0.01$. 


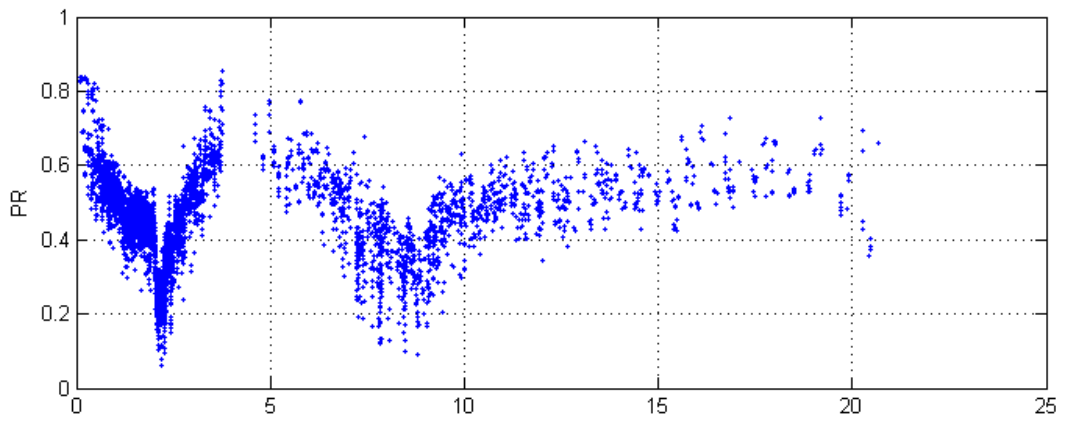

(a)

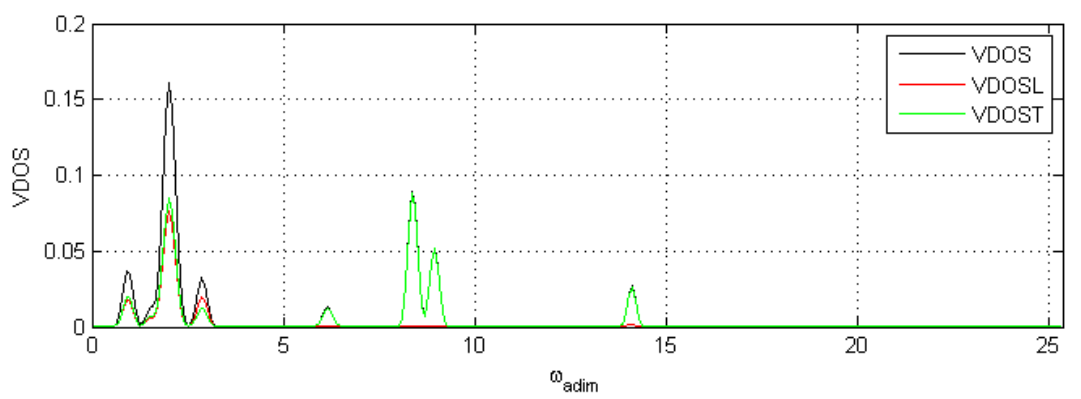

(b)

Figure 19: (a) PR and (b) complete, longitudinal and transverse VDOS of the isolated star, for the $4^{\text {th }}$ approximant of the Octagonal Quasi-periodic tiling beam structure with $\frac{K_{v}}{K_{u}}=2$. 




(a)

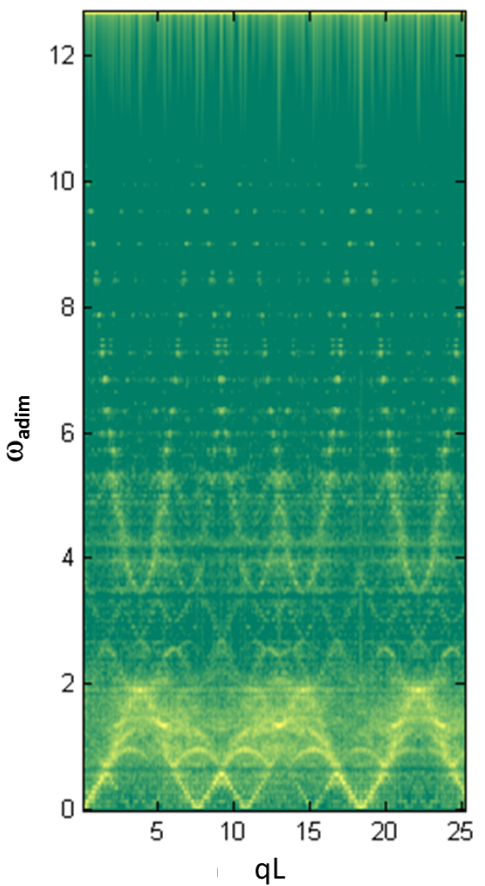

(b)

Figure 20: (a) Log of longitudinal DSF and (b) Log of transverse DSF for the $4^{\text {th }}$ approximant of the Octagonal Quasi-periodic tiling beam structure with $\frac{K_{v}}{K_{u}}=0.5$. 


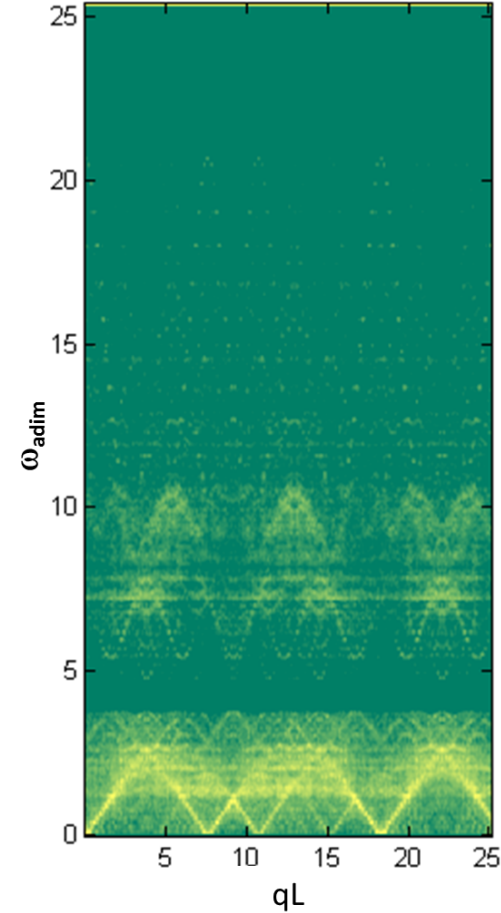

(a)

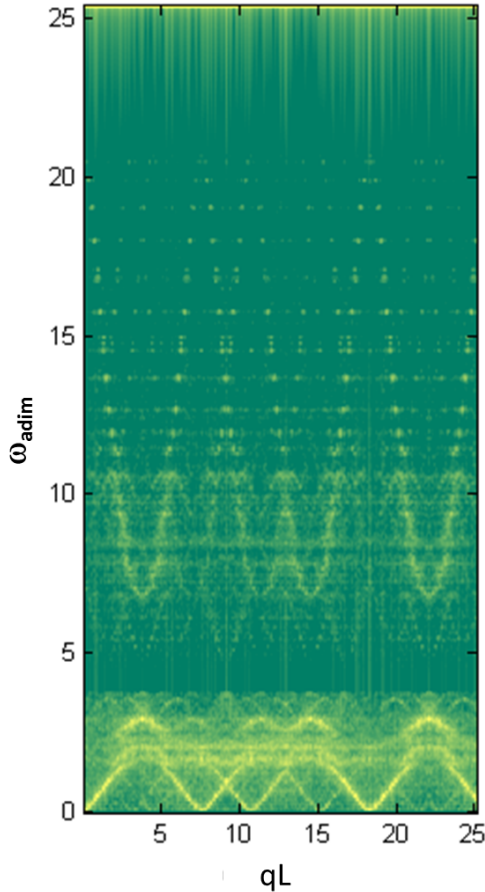

(b)

Figure 21: (a) Log of longitudinal DSF and (b) Log of transverse DSF for the $4^{\text {th }}$ approximant of the Octagonal Quasi-periodic tiling beam structure with $\frac{K_{v}}{K_{u}}=2$. 


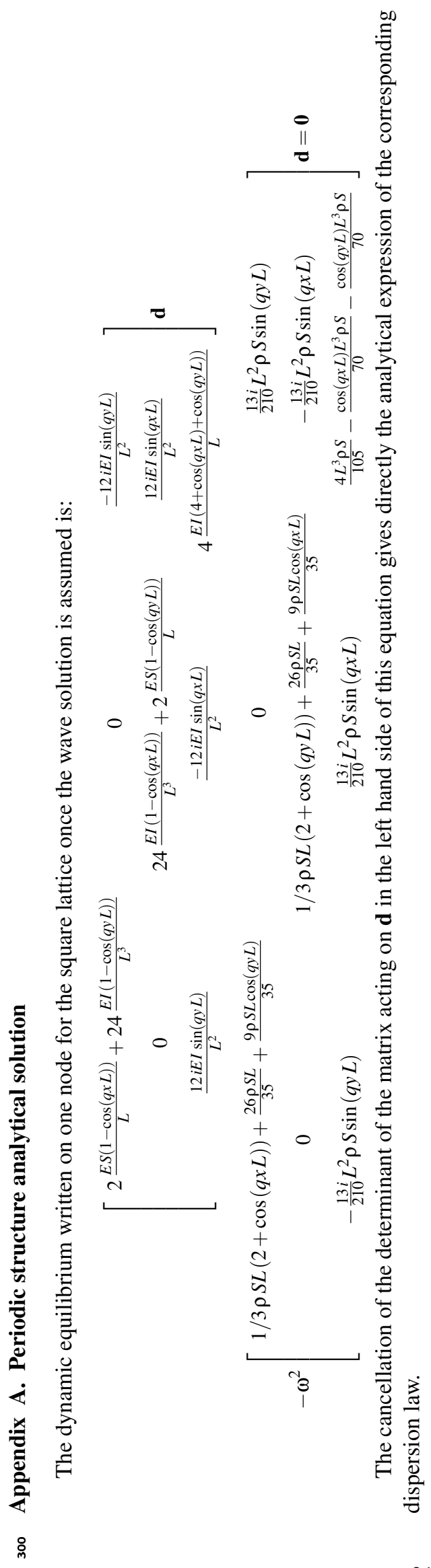




\section{References}

[1] J. Ashraff, R. Stinchcombe, Dynamic structure factor for the fibonacci-chain quasicrystal, Physical Review B 39 (4) (1989) 2670.

[2] Y. K. Vekilov, I. Gordeev, E. Isaev, Electronic spectrum of a two-dimensional fibonacci lattice, Journal of Experimental and Theoretical Physics 89 (5) (1999) 995999.

[3] A. Szallas, A. Jagannathan, Spin waves and local magnetizations on the penrose tiling, Physical Review B 77 (10) (2008) 104427.

[4] M. Bayindir, E. Cubukcu, I. Bulu, E. Ozbay, Photonic band-gap effect, localization, and waveguiding in the two-dimensional penrose lattice, Physical Review B 63 (16) (2001) 161104.

[5] M. Engel, S. Sonntag, H. Lipp, H.-R. Trebin, Structure factors of harmonic and anharmonic fibonacci chains by molecular dynamics simulations, Physical Review B 75 (14) (2007) 144203.

[6] M. Florescu, S. Torquato, P. J. Steinhardt, Complete band gaps in two-dimensional photonic quasicrystals, Physical Review B 80 (15) (2009) 155112.

[7] T. Bückmann, N. Stenger, M. Kadic, J. Kaschke, A. Frölich, T. Kennerknecht, C. Eberl, M. Thiel, M. Wegener, Tailored 3d mechanical metamaterials made by dip-in direct-laser-writing optical lithography, Advanced Materials 24 (20) (2012) 2710-2714.

[8] C. Claeys, B. Pluymers, P. Sas, W. Desmet, Design of a resonant metamaterial based acoustic enclosure, in: Proceedings of the 26th International Conference on Noise and Vibration Engineering, ISMA, 2014.

[9] C. C. Claeys, K. Vergote, P. Sas, W. Desmet, On the potential of tuned resonators to obtain low-frequency vibrational stop bands in periodic panels, Journal of Sound and Vibration 332 (6) (2013) 1418-1436.

[10] M. M. Sigalas, E. N. Economou, Elastic and acoustic wave band structure, Journal of sound and vibration 158 (2) (1992) 377-382.

[11] C. Chesnais, C. Boutin, S. Hans, Wave propagation and non-local effects in periodic frame materials: Generalized continuum mechanics, Mathematics and Mechanics of Solids 20 (8) (2015) 929-958.

[12] F. Dos Reis, J. Ganghoffer, Equivalent mechanical properties of auxetic lattices from discrete homogenization, Computational Materials Science 51 (1) (2012) 314-321.

[13] A. Sridhar, V. G. Kouznetsova, M. G. Geers, Homogenization of locally resonant acoustic metamaterials towards an emergent enriched continuum, Computational mechanics 57 (3) (2016) 423-435.

[14] H. Reda, J. Ganghoffer, H. Lakiss, Micropolar dissipative models for the analysis of 2d dispersive waves in periodic lattices, Journal of Sound and Vibration 392 (2017) $325-345$.

[15] S. de Rijk, E. Nijman, Equivalent material modelling of sandwich beams, evanescent solutions and damping investigations, Journal of Sound and Vibration 382 (2016) 291-309.

[16] M. Duneau, Approximants of quasiperiodic structures generated by the inflation mapping, Journal of Physics A: Mathematical and General 22 (21) (1989) 4549. 
[17] E. S. Sørensen, M. V. Jarić, M. Ronchetti, Ising model on penrose lattices: boundary conditions, Physical Review B 44 (17) (1991) 9271.

[18] L. Lin, Y. Saad, C. Yang, Approximating spectral densities of large matrices, SIAM Review 58 (1) (2016) 34-65.

[19] Y. Beltukov, C. Fusco, A. Tanguy, D. Parshin, Transverse and longitudinal vibrations in amorphous silicon, in: Journal of Physics: Conference Series, Vol. 661, IOP Publishing, 2016, p. 012056.

[20] H. Shang, R. Machado, J. Abdalla Filho, Dynamic analysis of euler-bernoulli beam problems using the generalized finite element method, Computers \& Structures 173 (2016) 109-122.

[21] H. Ozihel, Euler-Bernoulli Beam Equation, VDM Publishing, 2010. URL https://books.google.fr/books?id=6kpXXAACAAJ

[22] J. M. Larkin, A. J. McGaughey, Predicting alloy vibrational mode properties using lattice dynamics calculations, molecular dynamics simulations, and the virtual crystal approximation, Journal of Applied Physics 114 (2) (2013) 023507.

[23] S. Taraskin, S. Elliott, Nature of vibrational excitations in vitreous silica, Physical Review B 56 (14) (1997) 8605.

[24] J. Bauer, T.-M. Chang, J. Skinner, Correlation length and inverse-participation-ratio exponents and multifractal structure for anderson localization, Physical Review B 42 (13) (1990) 8121.

[25] P. Sheng, B. van Tiggelen, Introduction to Wave Scattering, Localization and Mesoscopic Phenomena, Springer, Berlin, 2006.

[26] Y. Beltukov, C. Fusco, D. Parshin, A. Tanguy, Boson peak and ioffe-regel criterion in amorphous siliconlike materials: The effect of bond directionality, Physical Review E 93 (2) (2016) 023006.

[27] A. Weisse, G. Wellein, A. Alvermann, H. Fehske, The kernel polynomial method, Reviews of Modern Physics 78 (2006) 275.

[28] N. Haskell, Total energy and energy spectral density of elastic wave radiation from propagating faults, Bulletin of the Seismological Society of America 54 (1969) 1811.

[29] V. M. Giordano, G. Monaco, Inelastic x-ray scattering study of liquid ga: Implications for the short-range order, Physical Review B 84 (5) (2011) 052201.

[30] J.-P. Boon, S. Yip, Molecular Hydrodynamics, McGraw-Hill, 1980.

[31] M. T. Dove, Introduction to lattice dynamics.

[32] J. Hafner, M. Krajčí, Propagating collective excitations in quasi-crystals, EPL (Europhysics Letters) 21 (1) (1993) 31.

[33] E. Baravelli, M. Ruzzene, Internally resonating lattices for bandgap generation and low-frequency vibration control, Journal of Sound and Vibration 332 (25) (2013) $6562-6579$.

[34] M. J. Leamy, Exact wave-based bloch analysis procedure for investigating wave propagation in two-dimensional periodic lattices, Journal of Sound and Vibration 331 (7) (2012) 1580-1596. 
[35] M. Duneau, R. Mosseri, C. Oguey, Approximants of quasiperiodic structures generated by the inflation mapping, Journal of Physics A 22 (1989) 4549.

390 [36] F. P. M. Beenker, Algebraic theory of non periodic tilings of the plane by two simple building blocks: a square and a rhombus, TH Report 82-WSK-04, Technische Hogeschool, Eindhoven, 1982.

[37] J. Hafner, M. Krajčí, M. Mihalkovič, Propagating and localized elementary excitations in decagonal quasicrystals, Physical review letters 76 (15) (1996) 2738.

395 [38] S. David, A. Chelnikov, J.-M. Lourtioz Duneau, IEEE Journal of Quantum Electronics Volume 37 (11) (2001) 1427.

[39] N. Auffray, R. Bouchet, Y. Brechet, Strain gradient elastic homogenization of bidimensional cellular media, International Journal of Solids and Structures 47 (13) (2010) 1698. 